\title{
Cyclic Sieving, Necklaces, and Branching Rules Related to Thrall's Problem
}

\author{
Connor Ahlbach * \\ Department of Mathematics \\ University of Washington, Seattle \\ Washington, U.S.A. \\ ahlbach@uw.edu
}

\author{
Joshua P. Swanson ${ }^{\dagger}$ \\ Department of Mathematics \\ University of California, San Diego \\ California, U.S.A. \\ jpswanson@ucsd.edu
}

Submitted: Sep 22, 2018; Accepted: Nov 7, 2018; Published: Nov 30, 2018

(C) The authors. Released under the CC BY-ND license (International 4.0).

\begin{abstract}
We show that the cyclic sieving phenomenon of Reiner-Stanton-White together with necklace generating functions arising from work of Klyachko offer a remarkably unified, direct, and largely bijective approach to a series of results due to KraśkiewiczWeyman, Stembridge, and Schocker related to the so-called higher Lie modules and branching rules for inclusions $C_{a} \imath S_{b} \hookrightarrow S_{a b}$. Extending the approach gives monomial expansions for certain graded Frobenius series arising from a generalization of Thrall's problem.
\end{abstract}

Mathematics Subject Classifications: 05E05, 05E10

\section{Introduction}

The Lie module $\mathcal{L}_{n}$ is the $n$th degree component of the free Lie algebra over $\mathbb{C}$ with $m$ generators, which is naturally a $\mathrm{GL}\left(\mathbb{C}^{m}\right)$-module. The Lie modules were famously studied by Thrall [Thr42] in the 1940's and have been extensively studied by Brandt [Bra44], Klyachko [Kly74], Kraśkiewicz-Weyman [KW01], Garsia [Gar90], Gessel-Reutenauer [GR93], Reutenauer [Reu93], Sundaram [Sun94], Schocker [Sch03], and many others. Thrall more generally introduced a certain GL( $\left.\mathbb{C}^{m}\right)$-decomposition $\oplus_{\lambda \in \operatorname{Par}} \mathcal{L}_{\lambda}$ of the tensor algebra of $\mathbb{C}^{m}$ arising from the Poincaré-Birkhoff-Witt theorem, where $\mathcal{L}_{(n)}=\mathcal{L}_{n}$. The $\mathcal{L}_{\lambda}$ are sometimes called the higher Lie modules. Thrall's original paper considered the determination of the multiplicity of the irreducible $V^{\mu}$ in $\mathcal{L}_{\lambda}$, which is often referred to as Thrall's problem. This problem is still open 75 years later. See Section 2.6 and [Reu93] for

*Partially supported by the National Science Foundation grant DMS-1101017.

${ }^{\dagger}$ Partially supported by the National Science Foundation grant DMS-1101017. 
more background on Thrall's problem and [Rei15] for a recent summary of related work. See Section 2 for missing definitions.

Kraśkiewicz-Weyman [KW01] gave a combinatorial solution to Thrall's problem when $\lambda=(n)$. In particular, they showed the multiplicity of $V^{\mu}$ in $\mathcal{L}_{(n)}$ is

$$
\#\left\{T \in \operatorname{SYT}(\mu): \operatorname{maj}(T) \equiv_{n} 1\right\},
$$

i.e. the number of standard tableaux of shape $\mu$ with major index 1 modulo $n$. Their argument crucially hinges upon the formula

$$
\operatorname{SYT}(\mu)^{\operatorname{maj}}\left(\omega_{n}^{r}\right)=\chi^{\mu}\left(\sigma_{n}^{r}\right)
$$

where we write the major index generating function as

$$
\operatorname{SYT}(\mu)^{\operatorname{maj}}(q):=\sum_{T \in \operatorname{SYT}(\mu)} q^{\operatorname{maj}(T)},
$$

$\omega_{n}$ is a primitive $n$th complex root of unity, $\sigma_{n}$ is an $n$-cycle in the symmetric group $S_{n}$, and $\chi^{\mu}$ is the character of the $S_{n}$-irreducible indexed by a partition $\mu$ of $n$. The analysis in [KW01] is somewhat indirect. It involves results of Lusztig and Stanley on coinvariant algebras and an intricate though beautiful argument involving $\ell$-decomposable partitions.

Equation (1) bears a striking resemblance to the cyclic sieving phenomenon (CSP) of Reiner-Stanton-White, which we now recall.

Definition 1.1. [RSW04] Suppose $C_{n}$ is a cyclic group of order $n$ generated by $\sigma_{n}, W$ is a finite set on which $C_{n}$ acts, and $f(q) \in \mathbb{Z}_{\geqslant 0}[q]$. We say the triple $\left(W, C_{n}, f(q)\right)$ exhibits the cyclic sieving phenomenon (CSP) if for all $r \in \mathbb{Z}$,

$$
\begin{aligned}
f\left(\omega_{n}^{r}\right) & =\# W^{\sigma_{n}^{r}} \\
& :=\#\left\{w \in W: \sigma_{n}^{r} \cdot w=w\right\}=\chi^{W}\left(\sigma_{n}^{r}\right),
\end{aligned}
$$

where $\omega_{n}$ is a primitive $n$th root of unity and $\chi^{W}$ is the character of $W$ as a $C_{n}$-module.

See [Sag11] for an excellent survey and introduction to cyclic sieving. The following cyclic sieving result also due to Reiner-Stanton-White is intimately related to (1). We use $W^{\text {stat }}(q)$ to denote $\sum_{w \in W} q^{\text {stat }(w)}$.

Theorem 1.2. [RSW04, Theorem 8.3, Proposition 4.4] Let $\alpha \vDash n$, let $\mathrm{W}_{\alpha}$ denote the set of all words of content $\alpha$, let $C_{n}$ act on $\mathrm{W}_{\alpha}$ by rotation, and let maj denote the major index statistic. Then, the triple

$$
\left(\mathrm{W}_{\alpha}, C_{n}, \mathrm{~W}_{\alpha}^{\mathrm{maj}}(q)\right)
$$

exhibits the CSP.

Since the sets $\mathrm{W}_{\alpha}$ are precisely the $S_{n}$-orbits for the natural $S_{n}$ action on length $n$ words, Theorem 1.2 may be thought of as a "universal sieving result" as follows. A very similar observation appeared in [BER11, Prop. 3.1]. 
Corollary 1.3. Let $W$ be a finite set of length $n$ words closed under the $S_{n}$-action. Then, the triple

$$
\left(W, C_{n}, W^{\mathrm{maj}}(q)\right)
$$

exhibits the CSP.

In [AS18], the authors introduced a new statistic on words, flex. As an example, flex $(221221)=2 \cdot 3=6$ since 221221 is the concatenation of 2 copies of the primitive word 221 and 221221 is third in lexicographic order amongst its 3 cyclic rotations. See Definition 2.3 for details. The flex statistic was designed to be "universal" for cyclic rather than symmetric actions on words in the following sense.

Lemma 1.4. [AS18, Lemma 8.3] Let $W$ be a finite set of length $n$ words closed under the $C_{n}$-action, where $C_{n}$ acts by cyclic rotations. Then, the triple

$$
\left(W, C_{n}, W^{\text {flex }}(q)\right)
$$

exhibits the CSP.

A corollary of these universal sieving results is the following equidistribution result. A more refined statement appeared in [AS18].

Theorem 1.5. [AS18, Theorem 8.4] Let $\mathrm{W}_{n}$ denote the set of length $n$ words, let maj $\mathrm{j}_{n}$ denote the major index modulo $n$ taking values in $\{1, \ldots, n\}$, and let cont denote the content of a word. We then have

$$
\mathrm{W}_{n}^{\text {cont, } \text { maj }_{n}}(\mathbf{x} ; q)=\mathrm{W}_{n}^{\text {cont,flex }}(\mathbf{x} ; q) .
$$

In Section 3, we show that the following well-known result of Kraśkiewicz-Weyman is essentially a corollary of Theorem 1.5. Here $\chi^{r}$ is the linear representation of the cyclic group $C_{n}$ given by $\chi^{r}\left(\sigma_{n}\right)=\omega_{n}^{r}$.

Theorem 1.6. [KW01] We have

$$
\operatorname{ch} \chi^{r} \uparrow_{C_{n}}^{S_{n}}=\sum_{\lambda \vdash n} a_{\lambda, r} s_{\lambda}(\mathbf{x})
$$

where

$$
a_{\lambda, r}:=\#\left\{Q \in \operatorname{SYT}(\lambda): \operatorname{maj}(Q) \equiv_{n} r\right\} .
$$

Klyachko [Kly74, Prop. 1] showed that the Lie modules $\mathcal{L}_{n}$ and the induced representations $\chi^{1 \uparrow S_{n}}$ are Schur-Weyl duals. The $\lambda=(n)$ case of Thrall's problem thus follows from Theorem 1.6 when $r=1$. More precisely, Klyachko expressed both the characteristic of $\chi^{1} \uparrow_{C_{n}}^{S_{n}}$ and the character of $\mathcal{L}_{n}$ as content generating functions on primitive necklaces of length $n$ words. We generalize this observation in Section 3 as follows, which also naturally motivates the flex statistic. 
Theorem 1.7. Let $\mathrm{NFD}_{n, r}$ denote the set of necklaces of length $n$ words with frequency dividing $r, \mathrm{~F}_{n, r}$ denote the set of length $n$ words with flex equal to $r$, and $\mathrm{M}_{n, r}(\mathbf{x})$ denote the set of length $n$ words with maj $_{n}$ equal to $r$. Then

$$
\operatorname{ch} \chi^{r \uparrow S_{n}}=\operatorname{NFD}_{n, r}^{\text {cont }}(\mathbf{x})=\mathrm{F}_{n, r}^{\text {cont }}(\mathbf{x})=\mathrm{M}_{n, r}^{\text {cont }}(\mathbf{x}) .
$$

Our new proof of Kraśkiewicz-Weyman's result reduces the problem of finding a bijective proof of a well-known symmetry result following from Theorem 1.6 to finding a bijective proof of the above equidistribution result, Theorem 1.5; see Corollary 3.5. It also provides a thus far rare example of an instance of cyclic sieving being used to prove other results rather than vice-versa.

In Section 4, we give a new proof of a result of Stembridge [Ste89] which settled a conjecture of Stanley describing the irreducible multiplicities of induced representations $\chi^{r} \uparrow_{\langle\sigma\rangle}^{S_{n}}$ for arbitrary $\sigma \in S_{n}$. The corresponding generalized major index statistics arise very naturally from the combinatorics of orbits and cyclic sieving.

In Section 5, we prove and generalize a result of Schocker [Sch03] concerning the higher Lie modules. Thrall's problem may be reduced to the $\lambda=\left(a^{b}\right)$ case by the LittlewoodRichardson rule. Bergeron-Bergeron-Garsia [BBG90] identified the Schur-Weyl dual of $\mathcal{L}_{\left(a^{b}\right)}$ as a certain induced module $\chi^{1,1 \uparrow S_{C_{a} S_{b}}}$ where $C_{a} \prec S_{b}$ is a wreath product; see Section 2.7 for details. Schocker gave a formula for the multiplicity of the irreducible $V^{\mu}$ in $\mathcal{L}_{\left(a^{b}\right)}$, though it involves many subtractions and divisions in general. We generalize Schocker's formula to all one-dimensional representations of $C_{a} \prec S_{b}$. In our approach, the subtractions and divisions in Schocker's formula arise naturally from the underlying combinatorics using Möbius inversion and Burnside's lemma.

The basic outline of each argument is the same: we obtain an orbit generating function from an explicit basis of a $\mathrm{GL}(V)$-module, we construct an appropriate necklace generating function, we use cyclic sieving to rewrite this generating function using words and descent statistics like the major index, and we finally apply RSK to get a Schur expansion. Transitioning from an orbit generating function to a necklace generating function where we can apply cyclic sieving involves various combinatorial techniques.

In Section 6, we discuss applying aspects of our approach to Thrall's problem in general. The arguments in the preceding sections strongly suggest attacking Thrall's problem by considering all branching rules for the inclusion $C_{a} \prec S_{b} \hookrightarrow S_{a b}$ rather than considering only one such rule. To that end, consider the irreducible representations $S^{\underline{\lambda}}$ of $C_{a} \prec S_{b}$, which are indexed by the set of $a$-tuples $\underline{\lambda}=\left(\lambda^{(1)}, \ldots, \lambda^{(a)}\right)$ of partitions with $\sum_{r=1}^{a}\left|\lambda^{(r)}\right|=b$. We first give the following plethystic expression for the corresponding characteristic.

Theorem 1.8. For all integers $a, b \geqslant 1$, we have

$$
\operatorname{ch} S^{\lambda} \uparrow_{C_{a} S_{a b}}^{S_{a b}}=\prod_{r=1}^{a} s_{\lambda^{(r)}}\left[\operatorname{NFD}_{a, r}^{\mathrm{cont}}(\mathbf{x})\right] .
$$

We then identify the analogues of the flex and $\mathrm{maj}_{n}$ statistics in this context, which send words to such $a$-tuples of partitions. We consequently give the following monomial expansion of the corresponding graded Frobenius series. See Section 2.7 and Section 6 for details. 
Theorem 1.9. Fix integers $a, b \geqslant 1$. We have

$$
\begin{aligned}
& \sum_{\underline{\lambda}} \operatorname{dim} S^{\underline{\lambda}} \cdot \operatorname{ch}\left(S^{\underline{\lambda} \uparrow} \uparrow_{C_{a} l S_{b}}^{S_{a b}}\right) q^{\underline{\lambda}}=\mathrm{W}_{a b}^{\text {cont,flex }}{ }^{b}(\mathbf{x} ; q) \\
& =\mathrm{W}_{a b}^{\mathrm{cont}, \mathrm{maj}_{a}^{b}}(\mathbf{x} ; q)
\end{aligned}
$$

where the sum is over all a-tuples $\underline{\lambda}=\left(\lambda^{(1)}, \ldots, \lambda^{(a)}\right)$ of partitions with $\sum_{r=1}^{a}\left|\lambda^{(r)}\right|=b$ and the $q^{\underline{\lambda}}$ are independent indeterminates.

The rest of the paper is organized as follows. In Section 2, we review combinatorial and representation-theoretic background. In particular, we summarize work related to Kraśkiewicz-Weyman's result, Theorem 1.6, in Section 2.5, and we discuss the current status of Thrall's problem in Section 2.6. In Section 3, we present our proof of KraśkiewiczWeyman's result, Theorem 1.6, using cyclic sieving. In Section 4, we give an analogous proof of Stembridge's result, Theorem 4.11. In Section 5, we give generalizations of Schocker's result, Theorem 5.11. In Section 6, we define the statistics $\operatorname{flex}_{a}^{b}$ and maj $_{a}^{b}$, prove Theorem 1.8 and Theorem 1.9, and discuss how the approach could be used to find the branching rules for $C_{a} \imath S_{b} \hookrightarrow S_{a b}$.

\section{Background}

Here we provide background on words, tableaux, Schur-Weyl duality, KraśkiewiczWeyman's result, Thrall's problem, and certain wreath products for use in later sections. All representations will be over $\mathbb{C}$. We write $[n]:=\{1, \ldots, n\}$, \#S for the cardinality of a set $S$, and

$$
\begin{aligned}
\left(\begin{array}{l}
S \\
k
\end{array}\right) & :=\{\text { all } k \text {-element subsets of } S\} \\
\left(\left(\begin{array}{l}
S \\
k
\end{array}\right)\right) & :=\{\text { all } k \text {-element multisubsets of } S\} .
\end{aligned}
$$

\section{$2.1 \quad$ Words}

We now recall standard combinatorial notions on words and fix some notation. A word $w$ of length $n$ is a sequence $w=w_{1} w_{2} \cdots w_{n}$ of letters $w_{i} \in \mathbb{Z}_{\geqslant 1}$. The descent set of $w$ is $\operatorname{Des}(w):=\left\{1 \leqslant i<n: w_{i}>w_{i+1}\right\}$. The major index of $w$ is $\operatorname{maj}(w):=\sum_{i \in \operatorname{Des}(w)} i$. Let $\operatorname{maj}_{n}(w)$ denote $\operatorname{maj}(w)$ modulo $n$ taking values in $[n]$.

The content of a word $w$, written $\operatorname{cont}(w)$, is the sequence $\alpha=\left(\alpha_{1}, \alpha_{2}, \ldots\right)$ where $\alpha_{j}$ is the number of $j$ 's in $w$. Such a sequence $\alpha$ is called a (weak) composition of $n$, written $\alpha \vDash n$. For $n \geqslant 1$ and $\alpha \vDash n$, we write the set of words of length $n$ or content $\alpha$ as

$$
\begin{aligned}
\mathrm{W}_{n} & :=\left\{w=w_{1} \cdots w_{n}: w_{i} \in \mathbb{Z}_{\geqslant 1}\right\}, \\
\mathrm{W}_{\alpha} & :=\left\{w \in \mathrm{W}_{n}: \operatorname{cont}(w)=\alpha\right\} .
\end{aligned}
$$


The set of all words with letters from $\mathbb{Z}_{\geqslant 1}$ is a monoid under concatenation. A word is primitive if it is not a power of a smaller word. Any non-empty word $w$ may be written uniquely as $w=v^{f}$ for $f \geqslant 1$ with $v$ primitive. The period of $w$, denoted $\operatorname{period}(w)$, is the length of $v$. The frequency of $w$, denoted freq $(w)$, is $f$.

The symmetric group $S_{n}$ acts on $\mathrm{W}_{n}$ by permuting the letters according to

$$
\sigma \cdot w_{1} w_{2} \cdots w_{n}:=w_{\sigma^{-1}(1)} w_{\sigma^{-1}(2)} \cdots w_{\sigma^{-1}(n)}
$$

for all $\sigma \in S_{n}$. In particular, letting $\sigma_{n}:=\left(\begin{array}{lll}1 & 2 & \cdots\end{array}\right) \in S_{n}$ and $C_{n}:=\left\langle\sigma_{n}\right\rangle$, the cyclic group $C_{n}$ acts on $\mathrm{W}_{n}$ by rotation according to

$$
\sigma_{n} \cdot w_{1} w_{2} \cdots w_{n}:=w_{n} w_{1} \cdots w_{n-1} .
$$

Definition 2.1. An orbit of $w \in \mathrm{W}_{n}$ under rotation is a necklace, denoted $[w]$. Note that $\operatorname{period}(w)=\#[w]$ and $\operatorname{freq}(w) \cdot \operatorname{period}(w)=n$. Content, primitivity, period, and frequency are all well-defined on necklaces. For $n \geqslant 1$, we write

$$
\mathrm{N}_{n}:=\{\text { necklaces of length } n \text { words }\} \text {. }
$$

Example 2.2. Consider $w=15531553 \in \mathrm{W}_{8}$. Then, the length of $w$ is $8, \operatorname{Des}(w)=$ $\{3,4,7\}, \operatorname{maj}(w)=14$, and $\operatorname{cont}(w)=(2,0,2,0,4)$, so $w \in \mathrm{W}_{(2,0,2,0,4)}$. Since $w=$ $15531553=(1553)^{2}$ and 1553 is primitive, $w$ is not primitive, $\operatorname{period}(w)=4$, and freq $(w)=2$. The necklace of $w$ is

$$
[w]=\{15531553,55315531,53155315,31553155\} \in \mathrm{N}_{8} .
$$

We now recall the flex statistic from [AS18].

Definition 2.3. Given $w \in \mathrm{W}_{n}$, let $\operatorname{lex}(w)$ denote the position at which $w$ appears in the lexicographic order of its rotations, starting at 1 . The flex statistic is given by

$$
\operatorname{flex}(w)=\operatorname{freq}(w) \cdot \operatorname{lex}(w) .
$$

Example 2.4. If $w=21132113$, its necklace is

$$
[w]=\{11321132,13211321,21132113,32113211\}
$$

listed in lexicographic order. Since $w$ is in the third position, $\operatorname{lex}(w)=3$. Here freq $(w)=2$, so $\operatorname{flex}(w)=6$.

\subsection{Generating Functions}

In most triples $\left(W, C_{n}, f(q)\right)$ that have been found to exhibit the CSP, $f(q)$ is a statistic generating function on $W$ for some well-known statistic. Given stat: $W \rightarrow \mathbb{Z}_{\geqslant 0}$, we write the corresponding generating function as

$$
W^{\text {stat }}(q):=\sum_{w \in W} q^{\text {stat }(w)}
$$


We use natural multivariable analogues of this notation as well. For example, letting $\mathbf{x}=\left(x_{1}, x_{2}, \ldots\right)$

$$
\mathrm{W}_{n}^{\text {cont,maj }}(\mathbf{x} ; q):=\sum_{w \in \mathrm{W}_{n}} \mathbf{x}^{\operatorname{cont}(w)} q^{\operatorname{maj}(w)} \in \mathbb{Z}_{\geqslant 0}\left[\left[x_{1}, x_{2}, \ldots\right]\right][q]
$$

where $\mathbf{x}^{\left(\alpha_{1}, \ldots, \alpha_{m}\right)}:=x_{1}^{\alpha_{1}} \cdots x_{m}^{\alpha_{m}}$.

\subsection{Tableaux}

A partition of $n$, denoted $\lambda \vdash n$, is a composition of $n$ whose parts weakly decrease. Write Par for the set of all partitions. The Young diagram of $\lambda$ is the upper-left justified collection of cells with $\lambda_{i}$ entries in the $i$ th row starting from the top. We may write a partition in exponential form as $\lambda=1^{m_{1}} 2^{m_{2}} \ldots \vdash n$ where $m_{i}$ is the number of parts of $\lambda$ of size $i$. In this case, the number of elements of $S_{n}$ with cycle type $\lambda$ is $\frac{n !}{z_{\lambda}}$ where $z_{\lambda}:=1^{m_{1}} 2^{m_{2}} \cdots m_{1} ! m_{2} ! \cdots$.

A semistandard Young tableau of shape $\lambda$ is a filling of the Young diagram of $\lambda$ with entries from $\mathbb{Z}_{\geqslant 1}$ which weakly increases along rows and strictly increases along columns. The set of semistandard Young tableaux of shape $\lambda$ is denoted $\operatorname{SSYT}(\lambda)$. The content of $P \in \operatorname{SSYT}(\lambda)$, denoted cont $(P)$, is the composition whose $j$-th entry is the number of $j$ 's in $P$. The set of standard Young tableaux of shape $\lambda$, denoted $\operatorname{SYT}(\lambda)$, is the subset of $\operatorname{SSYT}(\lambda)$ consisting of tableaux of content $(1, \ldots, 1) \vDash n$. The descent set of a tableau $Q \in \operatorname{SYT}(\lambda)$, denoted $\operatorname{Des}(Q)$, is the set of all $i \in[n-1]$ such that $i+1$ lies in a lower row of $Q$ than $i$.

Example 2.5. We draw our tableaux in English notation. The semistandard tableau

$$
P=\begin{array}{|l|l|l|l|l|l}
\hline 1 & 1 & 2 & 3 & 3 & 4 \\
\cline { 1 - 2 } 2 & 3 & 4 & 4 & 6 &
\end{array} \in \operatorname{SSYT}(6,5,1)
$$

has $\operatorname{cont}(P)=(2,2,4,3,0,1)$. The standard tableau

$$
Q=\begin{array}{|l|l|l}
\hline 1 & 2 & 5 \\
\hline 3 & 4 &
\end{array} \quad \in \operatorname{SYT}(3,2,1)
$$

has $\operatorname{Des}(Q)=\{2,5\}$, and $\operatorname{maj}(Q)=7$.

Let $\mathbf{x}=\left(x_{1}, x_{2}, \ldots\right)$. For a partition $\lambda$, the Schur function $s_{\lambda}$ is the content generating function on semistandard tableaux of shape $\lambda$,

$$
s_{\lambda}(\mathbf{x}):=\operatorname{SSYT}(\lambda)^{\operatorname{cont}}(\mathbf{x}):=\sum_{P \in \operatorname{SSYT}(\lambda)} \mathbf{x}^{\operatorname{cont}(P)} .
$$

The Schur functions are symmetric in the sense that they are unchanged under any permutation of the underlying variables. Two important instances of Schur functions are 
the complete homogeneous symmetric functions

$$
h_{n}(\mathbf{x}):=s_{(n)}(\mathbf{x})=\sum_{i_{1} \leqslant \cdots \leqslant i_{n}} x_{i_{1}} \cdots x_{i_{n}}
$$

and the elementary symmetric functions

$$
e_{n}(\mathbf{x}):=s_{\left(1^{n}\right)}(\mathbf{x})=\sum_{i_{1}<\cdots<i_{n}} x_{i_{1}} \cdots x_{i_{n}} .
$$

The power-sum symmetric functions are given by

$$
p_{n}(\mathbf{x}):=x_{1}^{n}+x_{2}^{n}+\cdots \quad \text { and } \quad p_{\left(\lambda_{1}, \ldots, \lambda_{k}\right)}(\mathbf{x}):=p_{\lambda_{1}}(\mathbf{x}) \cdots p_{\lambda_{k}}(\mathbf{x}) .
$$

Definition 2.6. The Robinson-Schensted-Knuth (RSK) correspondence is a bijection

$$
\begin{aligned}
\operatorname{RSK}: \mathrm{W}_{n} & \rightarrow \bigsqcup_{\lambda \vdash n} \operatorname{SSYT}(\lambda) \times \operatorname{SYT}(\lambda), \\
w & \mapsto(P(w), Q(w)) .
\end{aligned}
$$

The shape of $w$ under RSK, denoted $\operatorname{sh}(w)$, is the common shape of $P(w)$ and $Q(w)$. Two well-known properties of the RSK correspondence are

$$
\operatorname{cont}(w)=\operatorname{cont}(P(w)), \quad \operatorname{Des}(w)=\operatorname{Des}(Q(w)) .
$$

The fact that $\operatorname{Des}(w)=\operatorname{Des}(Q(w))$ is originally due to Schützenberger [Sch63, Remarque 2]. See [Sta99, Lemma 7.23.1] for a proof of (6) in the decisive permutation case and [Sta99, p.404] for further historical remarks. See [Sag01, Chapter 3] for more details on RSK.

We will repeatedly use the RSK correspondence to transition from the monomial to the Schur basis. These arguments all rely on the following result.

Lemma 2.7. Suppose $D \subset[n-1]$ and let

$$
\mathrm{W}_{n, D}:=\left\{w \in \mathrm{W}_{n}: \operatorname{Des}(w)=D\right\}
$$

be the set of length $n$ words with descent set $D$. For $\lambda \vdash n$, let

$$
a_{\lambda}^{D}:=\#\{Q \in \operatorname{SYT}(\lambda): \operatorname{Des}(Q)=D\} .
$$

Then

$$
\mathrm{W}_{n, D}^{\mathrm{cont}}(\mathbf{x})=\sum_{\lambda \vdash n} a_{\lambda}^{D} s_{\lambda}(\mathbf{x})
$$

Proof. Using RSK and (6), we have

$$
\begin{aligned}
\mathrm{W}_{n, D}^{\text {cont }}(\mathbf{x}) & =\left\{w \in \mathrm{W}_{n}: \operatorname{Des}(w)=D\right\}^{\operatorname{cont}}(\mathbf{x}) \\
& =\sum_{\lambda \vdash n} \sum_{\substack{Q \in \operatorname{SYT}(\lambda) \\
\operatorname{Des}(Q)=D}} \sum_{P \in \operatorname{SSYT}(\lambda)} x^{\operatorname{cont}(P)} \\
& =\sum_{\lambda \vdash n} \sum_{\substack{Q \in \operatorname{SYT}(\lambda) \\
\operatorname{Des}(Q)=D}} s_{\lambda}(\mathbf{x}) \\
& =\sum_{\lambda \vdash n} a_{\lambda}^{D} s_{\lambda}(\mathbf{x}) .
\end{aligned}
$$




\subsection{Schur-Weyl Duality}

We next summarize a few key points from the representation theory of $S_{n}$ and $\operatorname{GL}\left(\mathbb{C}^{m}\right)$. See [Ful97] for more.

The complex irreducible inequivalent representations of $S_{n}$ are canonically indexed by partitions $\lambda \vdash n$ and are called Specht modules, written $S^{\lambda}$. The Frobenius characteristic map ch is defined by $\operatorname{ch} S^{\lambda}:=s_{\lambda}(\mathbf{x})$ and is extended additively to all $S_{n}$-representations. Since Schur functions are $\mathbb{Z}$-linearly independent, computing the irreducible decomposition of an $S_{n}$-module $M$ corresponds to computing the Schur expansion of $\operatorname{ch} M$.

Let $V$ be a complex vector space of dimension $m$. Endow $V^{\otimes n}$ with the diagonal left GL $(V)$-action and the natural right $S_{n}$-action given by permutation of indexes. Given any $S_{n}$-module $M$, define a corresponding $\mathrm{GL}(V)$-module by

$$
E(M):=V^{\otimes n} \otimes_{\mathbb{C} S_{n}} M,
$$

which we call the Schur-Weyl dual of $M$. The irreducible inequivalent polynomial representations of GL $(V)$ are precisely the Schur Weyl duals of all $S^{\lambda}$ where $\lambda$ is a partition with at most $\operatorname{dim}(V)$ non-zero parts [Ful97, Thm. 8.2.2].

Let $E$ be a finite-dimensional, polynomial representation of $\operatorname{GL}(V)$ and pick a basis $\left\{v_{1}, \ldots, v_{m}\right\}$ for $V$. The Schur character of $E$, denoted ch $E$, is the trace of the action of $\operatorname{diag}\left(x_{1}, \ldots, x_{m}\right) \in \mathrm{GL}(V)$ on $E$, where the diagonal matrix is with respect to the basis $v_{1}, \ldots, v_{m}$. Polynomiality of $E$ implies $\operatorname{ch} E \in \mathbb{C}\left[x_{1}, \ldots, x_{m}\right]$. Moreover, $\operatorname{ch}(E)$ is a symmetric function of $x_{1}, \ldots, x_{m}$. In fact,

$$
\operatorname{ch} V^{\lambda}=\operatorname{ch} E\left(S^{\lambda}\right)=s_{\lambda}\left(x_{1}, \ldots, x_{m}, 0,0, \ldots\right) .
$$

Thus, for any $S_{n}$-module $M$, we have

$$
\lim _{m \rightarrow \infty} \operatorname{ch} E(M)=\operatorname{ch} M .
$$

In light of this, we often leave dependence on $m$ or $V$ implicit.

\subsection{Kraśkiewicz-Weyman Symmetric Functions}

The symmetric functions appearing in Theorem 1.5 have a wealth of important interpretations. Here we summarize some of these interpretations.

Definition 2.8. For $n \geqslant 1$, let

$$
\mathrm{KW}_{n}(\mathbf{x} ; q):=\sum_{\substack{\lambda \vdash n \\ r \in[n]}} a_{\lambda, r} s_{\lambda}(\mathbf{x}) q^{r}
$$

where $a_{\lambda, r}:=\#\left\{Q \in \operatorname{SYT}(\lambda): \operatorname{maj}(Q) \equiv_{n} r\right\}$. We call $\mathrm{KW}_{n}(\mathbf{x} ; q)$ the $n$th KraśkiewiczWeyman symmetric function.

These symmetric functions are intimately related to the irreducible representations of certain cyclic groups. 
Definition 2.9. Recall $\sigma_{n}:=(12 \cdots n) \in S_{n}$ and $C_{n}:=\left\langle\sigma_{n}\right\rangle \leqslant S_{n}$ be the cyclic group of order $n$ it generates. Fixing any primitive $n$th root of unity $\omega_{n}$, write the irreducible characters of $C_{n}$ as $\chi^{1}, \ldots, \chi^{n}$ where

$$
\chi^{r}\left(\sigma_{n}\right):=\omega_{n}^{r} .
$$

We sometimes write $\chi_{n}^{r}$ if we want to specify the cyclic group $C_{n}$ as well.

Theorem 1.6 gives our first interpretation of $\mathrm{KW}_{n}(\mathbf{x} ; q)$,

$$
\mathrm{KW}_{n}(\mathbf{x} ; q)=\sum_{r=1}^{n} \operatorname{ch} \chi^{r \uparrow S_{n}} q^{r} .
$$

Since the regular representation of $C_{n}$ is $\oplus_{r=1}^{n} \chi^{r}$, when $q=1$ the right-hand side of (8) is the Frobenius characteristic of the regular representation of $S_{n}$, denoted $\mathbb{C} S_{n}$. The right-hand side of (8) is hence similar to a graded Frobenius series for $\mathbb{C} S_{n}$ and tracks branching rules for the inclusion $C_{n} \hookrightarrow S_{n}$. By Theorem 1.7, we can also write this series as

$$
\mathrm{KW}_{n}(\mathbf{x} ; q)=\sum_{r=1}^{n} \operatorname{NFD}_{n, r}^{\text {cont }}(\mathbf{x}) q^{r} .
$$

Now consider the action of $\sigma_{n}$ on the $S_{n}$-irreducible $S^{\lambda}$. Since $\sigma_{n}^{n}=1 \in S_{n}$, the action of $\sigma_{n}$ on $S^{\lambda}$ is diagonal with eigenvalues $\omega_{n}^{k_{1}}, \omega_{n}^{k_{2}}, \ldots$ where $\omega_{n}$ is a fixed primitive $n$th root of unity and $1 \leqslant k_{i} \leqslant n$ for each $i$. Let $P_{\lambda}(q):=q^{k_{1}}+q^{k_{2}}+\cdots$ be the generating function of the cyclic exponents $k_{1}, k_{2}, \ldots$, which were studied extensively by Stembridge [Ste89]. Using the right-hand side of (8) and Frobenius reciprocity quickly gives the following.

Theorem 2.10 (See [Ste89, Prop. 1.2, Thm. 3.3]). The cyclic exponent generating function for $S_{n}$ is given by

$$
\mathrm{KW}_{n}(\mathbf{x} ; q)=\sum_{\lambda \vdash n} P_{\lambda}(q) s_{\lambda}(\mathbf{x}) .
$$

Next, extend the regular representation $\mathbb{C} S_{n}$ to an $S_{n} \times C_{n}$-module by letting $S_{n}$ act on the left and $C_{n}$ act on the right. There is a straightforward notion of an $S_{n} \times C_{n}$-Frobenius characteristic map given by sending an irreducible $S^{\lambda} \otimes \chi^{r}$ to $s_{\lambda}(\mathbf{x}) q^{r}$ where $q$ is an indeterminate satisfying $q^{n}=1$. The following now follows easily using the right-hand side of (8).

Corollary 2.11. [KW01] The $S_{n} \times C_{n}$-Frobenius characteristic of the regular representation is

$$
\mathrm{KW}_{n}(\mathbf{x} ; q)=\operatorname{ch}_{S_{n} \times C_{n}} \mathbb{C} S_{n} .
$$

It is well-known that the type $A_{n-1}$ coinvariant algebra $R_{n}$ is a graded $S_{n}$-module which is isomorphic as an ungraded $S_{n}$-module to $\mathbb{C} S_{n}$. We may give $R_{n}$ an $S_{n} \times C_{n}$-module structure by letting $C_{n}$ act on the $k$ th degree component of $R_{n}$ by $\sigma_{n} \cdot f:=\omega_{n}^{k} f$, where $\omega_{n}$ is a fixed primitive $n$th root of unity. Springer and, independently, Kraśkiewicz-Weyman showed that $\mathbb{C} S_{n}$ and $R_{n}$ are isomorphic as $S_{n} \times C_{n}$-modules. Consequently, from the right-hand side of (11), we have the following. 
Theorem 2.12 (Springer [Spr74, Prop. 4.5]; cf. [KW01, Thm. 1]). The $S_{n} \times C_{n}$-Frobenius characteristic of the coinvariant algebra $R_{n}$ is

$$
\mathrm{KW}_{n}(\mathbf{x} ; q)=\operatorname{ch}_{S_{n} \times C_{n}} R_{n}
$$

The graded Frobenius characteristic of the coinvariant algebra is the modified HallLittlewood symmetric function $\widetilde{Q}_{\left(1^{n}\right)}(\mathbf{x} ; q)$ [GP92, (I.8)]. Consequently, (12) gives

$$
\mathrm{KW}_{n}(\mathbf{x} ; q) \equiv \widetilde{Q}_{\left(1^{n}\right)}(\mathbf{x} ; q) \quad\left(\bmod q^{n}-1\right) .
$$

See also [Rho10, §3] for a nice summary of this connection.

We may instead use the right-hand side of (7) as a starting point. From Lemma 2.7, it follows that

$$
\mathrm{KW}_{n}(\mathbf{x} ; q)=\mathrm{W}_{n}^{\text {cont, }^{2} \mathrm{jaj}_{n}}(\mathbf{x} ; q) .
$$

From Theorem 1.5 and (14), our final interpretation of $\mathrm{KW}_{n}(\mathbf{x} ; q)$ in this subsection is

$$
\mathrm{KW}_{n}(\mathbf{x} ; q)=\mathrm{W}_{n}^{\text {cont,flex }}(\mathbf{x} ; q) .
$$

\subsection{Thrall's Problem}

We next define the Lie modules $\mathcal{L}_{\lambda}$ and summarize the status of Thrall's problem. See [Reu93] for more details.

The tensor algebra of $V$ is $T(V):=\oplus_{n=0}^{\infty} V^{\otimes n}$, which is naturally a graded GL $(V)$ representation. Let $\mathcal{L}(V)$ be the Lie subalgebra of $T(V)$ generated by $V$, called the free Lie algebra on $V$, so that $\mathcal{L}(V)$ is a graded $\mathrm{GL}(V)$-representation with graded components $\mathcal{L}_{n}(V)=V^{\otimes n} \cap \mathcal{L}(V)$ called Lie modules. The universal enveloping algebra $\mathfrak{U}(\mathcal{L}(V))$ is isomorphic to $T(V)$ itself. By the Poincaré-Birkhoff-Witt Theorem,

$$
\mathfrak{U}(\mathcal{L}(V)) \cong \bigoplus_{\lambda=1^{m_{1} 2^{m_{2}} \ldots}} \operatorname{Sym}^{m_{1}}\left(\mathcal{L}_{1}(V)\right) \otimes \operatorname{Sym}^{m_{2}}\left(\mathcal{L}_{2}(V)\right) \otimes \cdots
$$

as graded $\mathrm{GL}(V)$-representations, where the sum is over all partitions and $\operatorname{Sym}^{m}(M)$ is the $m$ th symmetric power of $M$ [Reu93, Lemma 8.22]. The higher Lie module associated to $\lambda=1^{m_{1}} 2^{m_{2}} \ldots$ is defined to be

$$
\mathcal{L}_{\lambda}(V):=\operatorname{Sym}^{m_{1}}\left(\mathcal{L}_{1}(V)\right) \otimes \operatorname{Sym}^{m_{2}}\left(\mathcal{L}_{2}(V)\right) \otimes \cdots .
$$

The Lie modules hence yield a $\mathrm{GL}(V)$-module decomposition $T(V) \cong \oplus_{\lambda \in \operatorname{Par}} \mathcal{L}_{\lambda}(V)$.

Thrall's problem is the determination of the multiplicity of $V^{\mu}$ in $\mathcal{L}_{\lambda}(V)$, for instance by counting explicit combinatorial objects. The well-known Littlewood-Richardson rule solves the analogous problem for $V^{\mu} \otimes V^{\nu}$. It follows from (16) and the Littlewood-Richardson rule that, for the purposes of Thrall's problem, we may restrict our attention to the case when $\lambda=\left(a^{b}\right)$ is a rectangle. Since

$$
\mathcal{L}_{\left(a^{b}\right)}(V)=\operatorname{Sym}^{b}\left(\mathcal{L}_{(a)}(V)\right)
$$


the single-row case is particularly fundamental.

Hall [Hal59, Lemma 11.2.1] introduced what is now called the Hall basis for $\mathcal{L}_{n}(V)$, which, in the $m \rightarrow \infty$ limit, is in content-preserving bijection with primitive necklaces $\mathrm{NFD}_{n, 1}$. For each primitive necklace, Hall associates a bracketing of its elements using what is now known as the Lyndon factorization [CFL58]. He gives an explicit, though computationally complex, algorithm to express any bracketing as a linear combination of the bracketings associated to primitive necklaces. Linear independence of these generators follows from a dimension count.

Klyachko consequently observed that the Schur character of $\mathcal{L}_{n}$ is the corresponding content generating function $\mathrm{NFD}_{n, 1}^{\text {cont }}(\mathbf{x})$. Taking symmetric powers, it follows that in the $m \rightarrow \infty$ limit, $\mathcal{L}_{\left(a^{b}\right)}(V)$ has a basis indexed by multisets of primitive necklaces and the Schur character is the following content generating function.

Lemma 2.13 (See [Kly74, Proposition 1]). We have, in the $m \rightarrow \infty$ limit,

$$
\operatorname{ch} \mathcal{L}_{(a)}=\operatorname{NFD}_{a, 1}^{\text {cont }}(\mathbf{x}) \quad \text { and } \quad \operatorname{ch} \mathcal{L}_{\left(a^{b}\right)}=\left(\left(\begin{array}{c}
\operatorname{NFD}_{a, 1} \\
b
\end{array}\right)\right)^{\text {cont }}(\mathbf{x}) .
$$

One formulation of Thrall's problem is hence to find the Schur expansion of the expressions in Lemma 2.13.

While we will not have direct need of it, we would be remiss if we did not mention the following beautiful and important result of Gessel and Reutenauer [GR93, (2.1)]. The expansion of $\operatorname{ch} \mathcal{L}_{\lambda}$ in terms of Gessel's fundamental quasisymmetric functions is

$$
\operatorname{ch} \mathcal{L}_{\lambda}=\sum_{\substack{\sigma \in S_{n} \\ \sigma \text { has cycle type } \lambda}} F_{n, \operatorname{Des}(\sigma)}(\mathbf{x})
$$

where

$$
F_{n, D}(\mathbf{x})=\sum_{\substack{i_{1} \leqslant \ldots \leqslant i_{n} \\ i_{j}<i_{j+1} \text { if } j \in D}} x_{i_{1}} \ldots x_{i_{n}} .
$$

Gessel and Reutenauer gave an elegant bijective proof of (18) in [GR93] involving multisets of primitive necklaces as in Lemma 2.13. Another formulation of Thrall's problem is thus to convert the right-hand side of (18) to the Schur basis.

Klyachko [Kly74] was the first to observe the intimate connections between Lie modules and the linear representations $\chi^{r}$ in Definition 2.9. Klyachko proved the $r=1$ case of Theorem 1.7, that $\operatorname{ch} \chi^{1} \uparrow_{C_{n}}^{S_{n}}=\operatorname{NFD}_{n, 1}^{\text {cont }}(\mathbf{x})$. Combining Klyachko's result, Lemma 2.13, the $r=1$ case of Theorem 1.7, and Kraśkiewicz-Weyman's result, Theorem 1.6, solves Thrall's problem when $\lambda=(n)$. Recall that if $\lambda \vdash n$, then $a_{\lambda, r}:=\#\left\{Q \in \operatorname{SYT}(\lambda): \operatorname{maj}(Q) \equiv_{n} r\right\}$.

Corollary 2.14. For all $\lambda \vdash n \geqslant 1$, the multiplicity of $V^{\lambda}$ in $\mathcal{L}_{(n)}$ is $a_{\lambda, 1}$.

Since $\chi^{r} \uparrow S_{C_{n}}$ depends up to isomorphism only on $n$ and $\operatorname{gcd}(n, r)$, we also have the following well-known symmetry.

Corollary 2.15. For all $\lambda \vdash n \geqslant 1$, we have $a_{\lambda, r}=a_{\lambda, \operatorname{gcd}(n, r)}$. 
Remark 2.16. A bijective proof of this symmetry is currently unknown.

Thrall's problem is an instance of a plethysm problem as we next describe. See [Sta99, Appendix 2] for more details. Given polynomial representations of general linear groups

$$
\rho: \mathrm{GL}(V) \rightarrow \mathrm{GL}(W) \quad \text { and } \quad \tau: \mathrm{GL}(W) \rightarrow \mathrm{GL}(X)
$$

where $V, W, X$ are finite-dimensional complex vector spaces, the plethysm of their Schur characters is the Schur character of their composite:

$$
(\operatorname{ch} \tau)[\operatorname{ch} \rho]:=\operatorname{ch}(\tau \circ \rho) .
$$

It is easy to see that $\operatorname{ch} \operatorname{Sym}^{b}(W)=h_{b}\left(x_{1}, \ldots, x_{m}\right)$ where $m=\operatorname{dim}(W)$. Consequently, (17) gives

$$
\operatorname{ch} \mathcal{L}_{\left(a^{b}\right)}=h_{b}\left[\operatorname{ch} \mathcal{L}_{a}\right] .
$$

Yet another formulation of Thrall's problem is thus to expand $h_{b}\left[\operatorname{ch} \mathcal{L}_{a}\right]$ in the Schur basis. Such plethysm problems are notoriously difficult. However, a combinatorial description for the Schur expansion of $\left(\operatorname{ch} \mathcal{L}_{a}\right)\left[h_{\nu}\right]$ in terms of the charge statistic was given by Lascoux-Leclerc-Thibon in [LLT94, Thm. 4.2] and [LLT97, Thm. III.3].

Remark 2.17. At present, Thrall's problem has only been solved in the following cases:

- when $\lambda=(n)$ has a single part (see Corollary 2.14);

- when $\lambda=\left(1^{n}\right), \mathcal{L}_{\left(1^{n}\right)}$ is the trivial representation;

- when $\lambda=\left(2^{b}\right), \operatorname{ch} \mathcal{L}_{\left(2^{b}\right)}=\sum s_{\mu}$ where the sum is over $\mu \vdash 2 b$ with even column sizes (see [Mac95, Ex. I.8.6(b), p. 138]).

\subsection{Wreath Products}

The Schur-Weyl duals of the higher Lie modules $\mathcal{L}_{\lambda}$ have also been identified in terms of induced representations of certain wreath products. Here we summarize this connection as well as some related aspects of the representation theory of wreath products which will be used in Section 6. Our presentation largely mirrors [Ste89].

Definition 2.18. Given a group $G$, the wreath product of $G$ with $S_{n}$, denoted $G$ 々 $S_{n}$, is the semidirect product explicitly described as follows. $G$ ? $S_{n}$ is the set $G^{n} \times S_{n}$ with multiplication given by

$$
\left(g_{1}, \ldots, g_{n}, \sigma\right) \cdot\left(h_{1}, \ldots, h_{n}, \tau\right):=\left(g_{1} h_{\sigma^{-1}(1)}, \ldots, g_{n} h_{\sigma^{-1}(n)}, \sigma \tau\right)
$$

for all $g_{1}, \ldots g_{n}, h_{1}, \ldots, h_{n} \in G$ and $\sigma, \tau \in S_{n}$. Furthermore, given $\alpha \vDash n$, set $G$ ? $\prod_{i} S_{\alpha_{i}}:=$ $\prod_{i}\left(G \succ S_{\alpha_{i}}\right)$, which has a natural inclusion into $G<S_{n}$. Roughly speaking, $G \succ S_{n}$ can be considered as the group of $n \times n$ "pseudo-permutation" matrices with entries from $G$. 
Now suppose $U$ is a $G$-set and $V$ is an $S_{n}$-set. There is a natural notion of $U$ ? $V$ as a

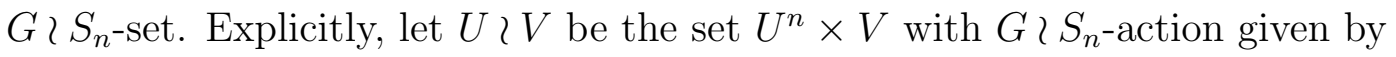

$$
\left(g_{1}, \ldots, g_{n}, \sigma\right) \cdot\left(u_{1}, \ldots, u_{n}, v\right):=\left(g_{1} \cdot u_{\sigma^{-1}(1)}, \ldots, g_{n} \cdot u_{\sigma^{-1}(n)}, \sigma \cdot v\right)
$$

for all $g_{1}, \ldots, g_{n} \in G, \sigma \in S_{n}, u_{1}, \ldots, u_{n} \in U, v \in V$. There is an analogous notion if $U$ is a $G$-module and $V$ is an $S_{n}$-module, namely $U \prec V:=U^{\otimes n} \otimes V$ with $G$ ? $S_{n}$-action

$$
\left(g_{1}, \ldots, g_{b}, \sigma\right) \cdot\left(u_{1} \otimes \cdots \otimes u_{b} \otimes v\right):=\left(g_{1} \cdot u_{\sigma^{-1}(1)}\right) \otimes \cdots \otimes\left(g_{b} \cdot u_{\sigma^{-1}(b)}\right) \otimes(\sigma \cdot v)
$$

extended $\mathbb{C}$-linearly.

Since $S_{a}$ acts naturally and faithfully on $[a],[a]<1_{b}$ has a natural $S_{a} \prec S_{b}$-action, where $1_{b}$ denotes the trivial $S_{b}$-set. Identifying $[a]<1_{b}$ with the set $[a b]$ and noting that the action remains faithful gives an inclusion $S_{a} \prec S_{b} \hookrightarrow S_{a b}$. Similarly we have an inclusion $C_{a} \prec S_{b} \hookrightarrow S_{a b}$. More concretely, $C_{a} \prec S_{b}$ acts faithfully on $[a b]$ by permuting the $b$ size- $a$ intervals in $[a b]$ amongst themselves and cyclically rotating each size- $a$ interval independently.

Remark 2.19. The induction product of two symmetric group representations corresponds to the product of their Frobenius characteristics, so that if $U$ is an $S_{a}$-module and $V$ is an $S_{b}$-module, then [Sta99, Prop. 7.18.2],

$$
\operatorname{ch}\left(U \otimes V \uparrow_{S_{a} \times S_{b}}^{S_{a+b}}\right)=(\operatorname{ch} U)(\operatorname{ch} V) .
$$

In Section 2.6, we considered the plethysm of Schur characters of general linear group representations. The corresponding operation for Frobenius characters of symmetric group representations is less well-known and involves wreath products as follows. Given two symmetric functions $f$ and $g=m_{1}+m_{2}+\cdots$ where the $m_{i}$ are all monomials, their plethysm is given by [Sta99, Def. A2.6]

$$
f[g]:=f\left(m_{1}, m_{2}, \ldots\right),
$$

which is well-defined since $f$ is symmetric. Then, if $U$ is an $S_{a}$-module and $V$ is an $S_{b}$-module, we have (see [Sta99, Thm. A2.8] or [Mac95, Appendix A, (6.2)])

$$
\operatorname{ch}\left((U \neg V) \uparrow_{S_{a} \backslash S_{b}}^{S_{a b}}\right)=\operatorname{ch}(V)[\operatorname{ch}(U)]
$$

When $G$ is a finite group, Specht [Spe32] described the complex inequivalent irreducible representations of $G<S_{n}$ in terms of those for $G$ and $S_{n}$, the conjugacy classes of $G$, and wreath products. In the case $C_{a} \prec S_{b}$, they are indexed by the following objects. 
Theorem 2.20 ([Spe32]; see [Ste89, Thm. 4.1]). The complex inequivalent irreducible representations of $C_{a} 2 S_{b}$ are indexed by a-tuples $\underline{\lambda}=\left(\lambda^{(1)}, \ldots, \lambda^{(a)}\right)$ of partitions with $\sum_{r=1}^{a}\left|\lambda^{(r)}\right|=b$. In particular, they are given by

$$
S^{\underline{\lambda}}:=\left(\left(\chi_{a}^{1} \prec S^{\lambda^{(1)}}\right) \otimes \cdots \otimes\left(\chi_{a}^{a} 2 S^{\lambda^{(a)}}\right)\right) \uparrow_{C_{a} 2 S_{\alpha(\underline{\lambda})} C_{a} S_{b}},
$$

where

$$
\begin{aligned}
\alpha(\underline{\lambda}) & :=\left(\left|\lambda^{(1)}\right|, \ldots,\left|\lambda^{(a)}\right|\right) \vDash b, \\
S_{\alpha(\underline{\lambda})} & :=S_{\left|\lambda^{(1)}\right|} \times \cdots \times S_{\left|\lambda^{(a)}\right|},
\end{aligned}
$$

$\chi_{a}^{r}$ is as defined in Definition 2.9, and $C_{a} 2 S_{\alpha(\underline{\lambda})}$ is viewed naturally as a subgroup of $C_{a} 2 S_{b}$.

One consequence of Theorem 2.20 is

$$
\operatorname{dim}\left(S^{\underline{\lambda}}\right)=\left(\begin{array}{c}
b \\
\alpha(\underline{\lambda})
\end{array}\right) \prod_{r=1}^{a} \# \operatorname{SYT}\left(\lambda^{(r)}\right) .
$$

Another consequence is an explicit description of the one-dimensional representations of $C_{a} \prec S_{b}$, which are as follows.

Definition 2.21. Fix integers $a, b \geqslant 1$. Let

$$
\chi^{r, 1}:=\chi_{a}^{r} \prec 1_{b} \quad \text { and } \chi^{r, \epsilon}:=\chi_{a}^{r} \prec \epsilon_{b}
$$

where $r=1, \ldots, a$ and $1_{b}$ and $\epsilon_{b}$ are the trivial and sign representations of $S_{b}$, respectively. When $b=1, \epsilon_{b}=1_{b}$, in which case $\chi^{r, 1}=\chi^{r, \epsilon}=\chi_{a}^{r}$. We sometimes write $\chi_{\left(a^{b}\right)}^{r, 1}$ or $\chi_{\left(a^{b}\right)}^{r, \epsilon}$ if we want to specify the group $C_{a} \prec S_{b}$ as well.

Bergeron-Bergeron-Garsia [BBG90] extended Klyachko's observation by showing that the Schur-Weyl dual of $\mathcal{L}_{\left(a^{b}\right)}$ is $\chi^{1,1} \uparrow_{C_{a} S_{a b} S_{b}}$. We next give a different argument of this fact which is straightforward given the preceding background and which uses a lemma we will require later in Section 6.

Lemma 2.22. We have

$$
\operatorname{ch} \chi^{1,1 \uparrow S_{a b} S_{a} S_{b}}=\left(\left(\begin{array}{c}
\mathrm{NFD}_{a, 1} \\
b
\end{array}\right)\right)^{\mathrm{cont}}(\mathbf{x}) .
$$

Proof. By Lemma 2.25 below and the fact that $C_{a} \curlywedge S_{b} \subseteq S_{a} \curlywedge S_{b} \subseteq S_{a b}$, we have

$$
\chi^{1,1 \uparrow \uparrow_{C_{a} l S_{b}}^{S_{a b}}}=\left(\chi_{a}^{1} 21_{b}\right) \uparrow_{C_{a} \imath S_{b}}^{S_{a b}} \cong\left(\chi_{a}^{1 \uparrow S_{C_{a}}^{S_{a}}} 21_{b}\right) \uparrow_{S_{a} \imath S_{b}}^{S_{a b}} .
$$

By (22) and the $r=1$ case of Theorem 1.7,

$$
\operatorname{ch}\left(\chi_{a}^{1} \uparrow_{C_{a}}^{S_{a}} \prec 1_{b}\right) \uparrow_{S_{a} S_{b}}^{S_{a b}}=\left(\operatorname{ch} 1_{b}\right)\left[\operatorname{ch} \chi_{a}^{1} \uparrow_{C_{a}}^{S_{a}}\right]=h_{b}\left[\operatorname{NFD}_{a, 1}^{\operatorname{cont}}(\mathbf{x})\right],
$$

since $\operatorname{ch}\left(1_{b}\right)=h_{b}(\mathbf{x})$. Now, $h_{b}\left[\mathrm{NFD}_{a, 1}^{\text {cont }}(\mathbf{x})\right]=\left(\left(\begin{array}{c}\mathrm{NFD}_{a, 1} \\ b\end{array}\right)\right)^{\text {cont }}(\mathbf{x})$ from the definition of plethysm, (21), and the definition of $h_{b}$, (4). The result will be complete once we prove Lemma 2.25. 
Corollary 2.23 ([BBG90, §4.4]; see also [Reu93, Thm. 8.24]). The Schur-Weyl dual of $\mathcal{L}_{\left(a^{b}\right)}$ is $\chi_{a}^{1 \uparrow} \uparrow_{C_{a} i S_{b}}^{S_{a b}}$.

Proof. Combine Lemma 2.13 and Lemma 2.22.

Indeed, the Schur-Weyl duals of general $\mathcal{L}_{\lambda}$ can be expressed very explicitly in terms of induced linear representations as follows. Suppose $\sigma \in S_{n}$ has cycle type $\lambda$. Write $Z_{\lambda}$ for the centralizer of $\sigma$ in $S_{n}$. When $\lambda=\left(a^{b}\right)$, it is straightforward to see that $Z_{\left(a^{b}\right)} \cong C_{a} \curlywedge S_{b}$. Furthermore, when $\lambda=1^{b_{1}} 2^{b_{2}} \cdots k^{b_{k}}$ is written in exponential notation, we have $Z_{\lambda} \cong Z_{\left(1^{b_{1}}\right)} \times Z_{\left(2^{b_{2}}\right)} \times \cdots \times Z_{\left(k^{b_{k}}\right)}$.

Corollary 2.24 (see [Reu93, Thm. 8.24]). Suppose $\lambda=1^{b_{1}} 2^{b_{2}} \cdots k^{b_{k}} \vdash n$. Let $\chi_{\lambda}^{1,1}$ denote the linear representation of $Z_{\lambda} \leqslant S_{n}$ given by the (outer) tensor product of the representations $\chi_{\left(i^{b_{i}}\right)}^{1,1}$ of $C_{i} 2 S_{b_{i}}$ for $1 \leqslant i \leqslant k$. Then, the Schur-Weyl dual of $\mathcal{L}_{\lambda}$ is $\chi_{\lambda}^{1,1} \uparrow_{Z_{\lambda}}^{S_{n}}$.

Proof. Using in order (16), multiplicativity of Schur characters under tensor products, Corollary 2.23, (20), Lemma 2.26 and transitivity of induction, the fact that $Z_{\lambda} \cong$ $\prod_{i=1}^{k} Z_{\left(i^{b_{i}}\right)}$, and the definition of $\chi_{\lambda}^{1,1}$, we have

$$
\begin{aligned}
\operatorname{ch} \mathcal{L}_{\lambda} & =\operatorname{ch}\left(\bigotimes_{i=1}^{k} \mathcal{L}_{\left(i^{b} i\right)}\right)=\prod_{i=1}^{k} \operatorname{ch} \mathcal{L}_{\left(i^{b_{i}}\right)}=\prod_{i=1}^{k} \operatorname{ch} \chi_{\left(i^{b_{i}}\right)}^{1,1} \uparrow_{Z_{\left(i^{b} b_{i}\right)}}^{S_{i b_{i}}} \\
& =\operatorname{ch}\left(\bigotimes_{i=1}^{k} \chi_{\left(i^{b_{i}}\right)}^{1,1} \uparrow_{Z_{\left(i^{b_{i}}\right)}}^{S_{i b_{i}}}\right) \uparrow_{S_{1 b_{1}} \times S_{2 b_{2}} \times \cdots}^{S_{n}}=\operatorname{ch}\left(\bigotimes_{i=1}^{k} \chi_{\left(i^{b_{i}}\right)}^{1,1}\right) \uparrow_{Z_{\lambda}}^{S_{n}} \\
& =\operatorname{ch} \chi_{\lambda}^{1,1 \uparrow \uparrow_{Z_{\lambda}}^{S_{n}}}
\end{aligned}
$$

The result will be complete once we prove Lemma 2.26.

Lemma 2.25. Suppose that $H$ is a subgroup of a group $G$, that $U$ is an $H$-module, and that $V$ is an $S_{n}$-module. Then

$$
(U<V) \uparrow_{H\left(S_{n}\right.}^{G i S_{n}} \cong\left(U \uparrow \uparrow_{H}^{G}\right)<V
$$

as $G \backslash S_{n}$-modules.

Proof. As sets, we have

$$
\begin{aligned}
(U \succ V) \uparrow \uparrow_{H\left(S_{n}\right.}^{G i S_{n}} & =\mathbb{C}\left(G \succ S_{n}\right) \otimes_{\mathbb{C}\left(H \imath S_{n}\right)}\left(U^{\otimes n} \otimes V\right), \\
\left(U \uparrow \uparrow_{H}^{G}\right) \curlywedge V & =\left(\mathbb{C} G \otimes_{\mathbb{C} H} U\right)^{\otimes n} \otimes V .
\end{aligned}
$$

Define

$$
\begin{aligned}
& \phi:(U \prec V) \uparrow_{H\left(S_{n}\right.}^{G i S_{n}} \rightarrow\left(U \uparrow_{H}^{G}\right) \prec V, \\
& \psi:\left(U \uparrow \uparrow_{H}^{G}\right)<V \rightarrow(U<V) \uparrow_{H\left(S_{n}\right.}^{G i S_{n}}
\end{aligned}
$$


by

$$
\begin{aligned}
\phi\left(\left(g_{1}, \ldots, g_{n}, \tau\right) \otimes\left(u_{1} \otimes \cdots \otimes u_{n} \otimes v\right)\right) \\
\quad:=\left(g_{1} \otimes u_{\tau^{-1}(1)}\right) \otimes \cdots \otimes\left(g_{n} \otimes u_{\tau^{-1}(n)}\right) \otimes(\tau \cdot v), \\
\psi\left(\left(g_{1} \otimes x_{1}\right) \otimes \cdots \otimes\left(g_{n} \otimes x_{n}\right) \otimes y\right) \\
\quad:=\left(g_{1}, \ldots, g_{n}, 1\right) \otimes\left(x_{1} \otimes \cdots \otimes x_{n} \otimes y\right)
\end{aligned}
$$

extended $\mathbb{C}$-linearly. It is straightforward to check directly that $\phi$ and $\psi$ are well-defined, $G \prec S_{n}$-equivariant, and mutual inverses. Note that showing $\psi \circ \phi(x)=x$ requires using the relation

$$
\left(g_{1}, \ldots, g_{n}, \tau\right) \otimes z=\left(g_{1}, \ldots, g_{n}, 1\right) \otimes(\tau \cdot z)
$$

in $\mathbb{C}\left(G / S_{n}\right) \otimes_{\mathbb{C}\left(H\left\langle S_{n}\right)\right.}\left(U^{\otimes n} \otimes V\right)$ for $g_{1}, \ldots, g_{n} \in G, \tau \in S_{n}, z \in U^{\otimes n} \otimes V$.

Lemma 2.26. Suppose that $H_{1}, \ldots, H_{k}$ are subgroups of groups $G_{1}, \ldots, G_{k}$ and that $U_{i}$ is an $H_{i}$-module for $1 \leqslant i \leqslant k$. Then

$$
\left(U_{1} \otimes \cdots \otimes U_{k}\right) \uparrow \uparrow_{H_{1} \times \cdots \times H_{k}}^{G_{1} \times \cdots \times G_{k}} \cong U_{1} \uparrow_{H_{1}}^{G_{1}} \otimes \cdots \otimes U_{k} \uparrow_{H_{k}}^{G_{k}}
$$

as $G_{1} \times \cdots \times G_{k}$-modules.

Proof. Having chosen bases for both sides, there is a natural $\mathbb{C}$-linear map between them. It is easy to check this is also $G_{1} \times \cdots \times G_{k}$-equivariant. The details are omitted.

\section{Cyclic Sieving and Kraśkiewicz-Weyman's Result}

In this section, we first build on work of Klyachko to prove Theorem 1.7. We then recover Kraśkiewicz-Weyman's result, Theorem 1.6, and discuss some benefits of our approach.

Klyachko observed in [Kly74, Prop. 1] that $E\left(\chi^{1 \uparrow \uparrow_{C_{n}}^{S_{n}}}\right)$, like $\mathcal{L}_{(n)}$, also has a basis indexed by primitive necklaces. Klyachko's argument may be readily generalized to $E\left(\chi^{r \uparrow S_{n}} C_{n}\right)$ as follows. Recall from the introduction that

$$
\begin{aligned}
\operatorname{NFD}_{n, r} & :=\left\{N \in \mathrm{N}_{n}: \operatorname{freq}(N) \mid r\right\}, \\
\mathrm{F}_{n, r} & :=\left\{w \in \mathrm{W}_{n}: \operatorname{flex}(w)=r\right\}, \\
\mathrm{M}_{n, r} & :=\left\{w \in \mathrm{W}_{n}: \operatorname{maj}_{n}(w)=r\right\} .
\end{aligned}
$$

In particular, $\mathrm{NFD}_{n, n}=\mathrm{N}_{n}$, and $\mathrm{NFD}_{n, 1}$ is the set of primitive necklaces of length $n$.

Theorem 3.1. There is a basis for $E\left(\chi^{r} \uparrow S_{C_{n}}\right)$ indexed by necklaces of length $n$ words with letters from $[\mathrm{m}]$ and with frequency dividing $r$. Moreover,

$$
\operatorname{ch} \chi^{r} \uparrow_{C_{n}}^{S_{n}}=\operatorname{NFD}_{n, r}^{\text {cont }}(\mathbf{x})
$$


Proof. Suppose the underlying vector space $V$ has basis $\left\{v_{1}, \ldots, v_{m}\right\}$. By a slight abuse of notation, we may view $\chi^{r}$ as the vector space $\mathbb{C}$ with the left $C_{n}$-action $\sigma_{n} \cdot 1:=\omega_{n}^{r}$. Since $\chi^{r \uparrow S_{n}^{S_{n}}}:=\mathbb{C} S_{n} \otimes_{\mathbb{C} C_{n}} \chi^{r}$, we have

$$
E\left(\chi^{r} \uparrow_{C_{n}}^{S_{n}}\right)=V^{\otimes n} \otimes_{\mathbb{C} S_{n}} \mathbb{C} S_{n} \otimes_{\mathbb{C} C_{n}} \chi^{r} \cong V^{\otimes n} \otimes_{\mathbb{C} C_{n}} \chi^{r}
$$

where $C_{n}$ acts on $V^{\otimes n}$ on the right by "rotating" the components of simple tensors. A spanning set for $V^{\otimes n} \otimes_{\mathbb{C} C_{n}} \chi^{r}$ is given by all $v_{i_{1}} \otimes \cdots \otimes v_{i_{n}} \otimes 1$, which we abbreviate as $\left[\begin{array}{lll}i_{1} & \cdots & i_{n}\end{array}\right]$. Acting by $\sigma_{n}^{-1}$ on $\chi^{r}$ on the left or on $V^{\otimes n}$ on the right gives the relation

$$
\left[i_{1} \cdots i_{n}\right]=\omega_{n}^{r}\left[i_{2} \cdots i_{n} i_{1}\right] .
$$

This relation shows that $\left[i_{1} \cdots i_{n}\right]$ is well-defined on the level of necklaces, at least up to nonzero scalar multiplication, which explains our notation. If the word $i_{1} \cdots i_{n}$ has frequency $f$ and period $p$, we then find

$$
\begin{aligned}
{\left[\begin{array}{lll}
i_{1} & \cdots & i_{n}
\end{array}\right] } & =\frac{1}{n} \sum_{j=0}^{n-1} \omega_{n}^{j r}\left[i_{j+1} \cdots i_{n} i_{1} \cdots i_{j}\right] \\
& =\frac{1}{n} \sum_{k=0}^{p-1}\left(\sum_{\ell=0}^{f-1} \omega_{n}^{(\ell p+k) r}\right)\left[i_{k+1} \cdots i_{n} i_{1} \cdots i_{k}\right] \\
& =\frac{1}{n}\left(\sum_{\ell=0}^{f-1} \omega_{n}^{\ell p r}\right) \sum_{k=0}^{p-1} \omega_{n}^{k r}\left[i_{k+1} \cdots i_{n} i_{1} \cdots i_{k}\right] .
\end{aligned}
$$

Since $\omega_{n}^{p}$ is a primitive $n / p=f$-th root of unity, the factor $\sum_{\ell=0}^{f-1} \omega_{n}^{\ell p r}$ is nonzero if and only if $\omega_{n}^{p r}=1$, so if and only if $f \mid r$. Picking representatives for necklaces with frequency dividing $r$ thus gives a spanning set for $E\left(\chi^{r \uparrow S_{n}}\right)$, and it is easy to see it is in fact a basis. Diagonal matrices act on this basis via

$$
\operatorname{diag}\left(x_{1}, \ldots, x_{n}\right) \cdot\left[i_{1} \cdots i_{n}\right]=\mathbf{x}^{\operatorname{cont}\left(i_{1} \cdots i_{n}\right)}\left[i_{1} \cdots i_{n}\right],
$$

from which it follows that the Schur character is the content generating function of necklaces of length $n$ words with letters from $[m]$ and with frequency dividing $r$. Letting $m \rightarrow \infty$, (27) follows.

Lemma 3.2. We have

$$
\operatorname{NFD}_{n, r}^{\text {cont }}(\mathbf{x})=\mathrm{F}_{n, r}^{\text {cont }}(\mathbf{x})=\mathrm{M}_{n, r}^{\text {cont }}(\mathbf{x})
$$

Proof. Consider the map

$$
\begin{aligned}
\iota: \mathrm{F}_{n, r} & \rightarrow \mathrm{NFD}_{n, r} \\
\iota(w) & :=[w] .
\end{aligned}
$$


Since flex $(w)=\operatorname{freq}(w) \operatorname{lex}(w)=r$, we have freq $(w) \mid r$, so $[w] \in \operatorname{NFD}_{n, r}$. Thus, $\iota$ is in fact a map from $\mathrm{F}_{n, r}$ to $\mathrm{NFD}_{n, r}$. Since each necklace in $\mathrm{NFD}_{n, r}$ contains exactly one word with flex equal to $r, \iota$ is a content-preserving bijection. Therefore, $\operatorname{NFD}_{n, r}^{\text {cont }}(\mathbf{x})=\mathrm{F}_{n, r}^{\text {cont }}(\mathbf{x})$.

Using Theorem 1.5, we have

$$
\mathrm{W}_{n}^{\text {cont,flex }}(\mathbf{x} ; q)=\mathrm{W}_{n}^{\text {cont }^{\text {maj }}}{ }_{n}(\mathbf{x} ; q),
$$

which means $\mathrm{F}_{n, r}^{\mathrm{cont}}(\mathbf{x})=\mathrm{M}_{n, r}^{\mathrm{cont}}(\mathbf{x})$.

Remark 3.3. From Theorem 3.1 and Lemma 3.2, the Schur character of $\chi^{r} \uparrow_{C_{n}}^{S_{n}}$ may be described as a content generating function for certain necklaces or for certain words. This proves Theorem 1.7 from the introduction.

We may now present our remarkably direct proof of Kraśkiewicz-Weyman's result, Theorem 1.6, using cyclic sieving.

Proof (of Theorem 1.6). The argument in Theorem 3.1 exhibited an explicit basis of the Schur module $E\left(\chi^{r \uparrow S_{n}}\right)$, showing that

$$
\sum_{r=1}^{n} \operatorname{ch} \chi^{r} \uparrow_{C_{n}}^{S_{n}} q^{r}=\sum_{r=1}^{n} \operatorname{NFD}_{n, r}^{\text {cont }}(\mathbf{x}) q^{r} .
$$

From Lemma 3.2, the bijection $\iota: \mathrm{F}_{n, r} \stackrel{\sim}{\rightarrow} \mathrm{NFD}_{n, r}$ given by $w \mapsto[w]$ gives

$$
\sum_{r=1}^{n} \operatorname{NFD}_{n, r}^{\text {cont }}(\mathbf{x}) q^{r}=\mathrm{W}_{n}^{\text {cont,flex }}(\mathbf{x} ; q) .
$$

Using universal cyclic sieving on words for $S_{n}$-orbits and $C_{n}$-orbits as described in the introduction, Theorem 1.5 now gives

$$
\mathrm{W}_{n}^{\text {cont,flex }}(\mathbf{x} ; q)=\mathrm{W}_{n}^{\text {cont }^{\text {maj }}}{ }_{n}(\mathbf{x} ; q) .
$$

Using the RSK algorithm, Lemma 2.7 gives

$$
\mathrm{W}_{n}^{\text {cont, } \text { maj }_{n}}(\mathbf{x} ; q)=\sum_{\substack{\lambda \vdash n \\ r \in[n]}} a_{\lambda, r} s_{\lambda}(\mathbf{x}) q^{r} .
$$

Combining all of these equalities and extracting the coefficient of $q^{r}$ gives the result.

Every step of the preceding proof uses an explicit bijection with the exception of the appeal to cyclic sieving through Theorem 1.5. This suggests the problem of finding a bijective proof of Theorem 1.5.

Problem 3.4. For each $n \geqslant 1$, find an explicit, content-preserving bijection

$$
\phi: \mathrm{W}_{n} \rightarrow \mathrm{W}_{n}
$$

such that $\operatorname{maj}_{n}(w)=\operatorname{flex}(\phi(w))$. 
Corollary 3.5. A solution to Problem 3.4 would yield an explicit, bijective proof of the identity

$$
\sum_{\lambda \vdash n} a_{\lambda, r} s_{\lambda}(\mathbf{x})=\sum_{\lambda \vdash n} a_{\lambda, s} s_{\lambda}(\mathbf{x})
$$

for any $r, s \in \mathbb{Z}$ where $\operatorname{gcd}(n, r)=\operatorname{gcd}(n, s)$.

Proof. We have content-preserving bijections

$$
\bigsqcup_{\lambda \vdash n} \operatorname{SSYT}(\lambda) \times\left\{Q \in \operatorname{SYT}(\lambda): \operatorname{maj}(Q) \equiv_{n} r\right\} \stackrel{\text { RSK }}{\rightarrow} \mathrm{M}_{n, r} \stackrel{\phi}{\rightarrow} \mathrm{F}_{n, r} \stackrel{\iota}{\rightarrow} \mathrm{NFD}_{n, r} .
$$

Now note that

$$
\mathrm{NFD}_{n, r}=\mathrm{NFD}_{n, \operatorname{gcd}(n, r)}=\mathrm{NFD}_{n, \operatorname{gcd}(n, s)}=\mathrm{NFD}_{n, s}
$$

We thus have an explicit, content-preserving bijection

$$
\begin{aligned}
\bigsqcup_{\lambda \vdash n} \operatorname{SSYT}(\lambda) & \times\left\{Q \in \operatorname{SYT}(\lambda): \operatorname{maj}(Q) \equiv_{n} r\right\} \\
& \stackrel{\sim}{\rightarrow} \bigsqcup_{\lambda \vdash n} \operatorname{SSYT}(\lambda) \times\left\{Q \in \operatorname{SYT}(\lambda): \operatorname{maj}(Q) \equiv_{n} s\right\}
\end{aligned}
$$

from which (31) follows.

Remark 3.6. The most difficult step in our proof of Theorem 1.6 is the universal $S_{n^{-}}$ cyclic sieving result, Corollary 1.3, or equivalently Theorem 1.2. The proof in [RSW04] of Theorem 1.2 perhaps unsurprisingly uses several of the interpretations of the KraśkiewiczWeyman symmetric functions from Section 2.5, in particular Theorem 2.12 involving $\mathrm{ch}_{S_{n} \times C_{n}} R_{n}$. However, both Kraśkiewicz-Weyman's and Springer's original proofs of Theorem 2.12 hinge upon (1). Indeed, Kraśkiewicz-Weyman showed explicitly in [KW01, Prop. 3] that $\operatorname{ch}_{S_{n} \times C_{n}} \mathbb{C} S_{n}=\operatorname{ch}_{S_{n} \times C_{n}} R_{n}$ is easily equivalent to (1). Springer's argument proving (1) uses a Molien-style formula, while Kraśkiewicz-Weyman's argument uses a recursion involving $\ell$-cores and skew hooks.

One may thus ask about the relationship between (1) and the cyclic sieving result, Theorem 1.2. Using stable principal specializations, one can consider earlier approaches to have been "in the $s$-basis" and our approach to have been "in the $h$-basis" in the following sense. Let $\tau^{\lambda}$ be the $S_{n}$-character of $1 \uparrow S_{S_{\lambda}}$, which has $\operatorname{ch}\left(1 \uparrow S_{S_{\lambda}}\right)=h_{\lambda}$. We have

$$
\begin{aligned}
& \chi^{\lambda}\left(\sigma_{n}^{r}\right)=\operatorname{SYT}(\lambda)^{\mathrm{maj}}\left(\omega_{n}^{r}\right)=\left.(1-q) \cdots\left(1-q^{n}\right) s_{\lambda}\left(1, q, q^{2}, \ldots\right)\right|_{q=\omega_{n}^{r}}, \\
& \tau^{\lambda}\left(\sigma_{n}^{r}\right)=\mathrm{W}_{\lambda}^{\text {maj }}\left(\omega_{n}^{r}\right)=\left.(1-q) \cdots\left(1-q^{n}\right) h_{\lambda}\left(1, q, q^{2}, \ldots\right)\right|_{q=\omega_{n}^{r}} .
\end{aligned}
$$

where the first equality is (1), the second is [Sta99, Prop. 7.19.11], the third is Theorem 1.2, and the fourth is [Sta99, Prop. 7.8.3] and [Mac13, Art. 6]. Our approach suggests that, as far as the Kraśkiewicz-Weyman theorem is concerned, the $h$-basis arises more directly.

In [AS18], the authors proved a refinement of Theorem 1.2. Since earlier approaches to Theorem 1.2 involving representation theory could not readily be adapted to this refinement, the argument instead uses completely different and highly combinatorial techniques. Thus, the arguments in [AS18] and the proof of Theorem 1.6 together give an essentially self-contained proof of Kraśkiewicz-Weyman's result. 


\section{Induced Representations of Arbitrary Cyclic Subgroups of $S_{n}$}

We next generalize the discussion in Section 3 to branching rules for general inclusions $\langle\sigma\rangle \hookrightarrow S_{n}$, recovering a result of Stembridge, Theorem 4.11. Following the outline of the previous section, we express the relevant characters in turn as a certain orbit generating function, Theorem 4.2, a necklace generating function, Lemma 4.6, and a generating function on words, Lemma 4.7. Two variations on the major index, $\mathbf{m a j} \mathbf{j}_{\nu}$ and $\mathbf{m a j}_{\nu}$, arise quite naturally from our argument. The CSP Theorem 1.2 again plays a decisive role.

Throughout this section, let $\sigma \in S_{n}$, let $C$ be the cyclic group generated by $\sigma$, and let $\ell:=\# C$ be the order of $\sigma$. Fixing a primitive $\ell$-th root of unity $\omega_{\ell}$, let $\chi^{r}: C \rightarrow \mathbb{C}$ for $r=1, \ldots, \ell$ be the linear $C$-module given by $\chi^{r}(\sigma):=\omega_{\ell}^{r}$. We begin by updating our notation for this setting and generalizing Theorem 3.1.

Definition 4.1. In analogy with Definition 2.1, suppose $\mathcal{O}$ is an orbit of $\mathrm{W}_{n}$ under the restricted $C$-action. The period of $\mathcal{O}$ is $\# \mathcal{O}$ and the frequency of $\mathcal{O}$, written freq $(\mathcal{O})$, is the stabilizer-order of any element of $\mathcal{O}$, or equivalently freq $(\mathcal{O})=\frac{\ell}{\# \mathcal{O}}$. The set of orbits of words whose frequency divides $r$ is

$$
\mathrm{OFD}_{C, r}:=\left\{C \text {-orbits } \mathcal{O} \text { of } \mathrm{W}_{n}: \operatorname{freq}(\mathcal{O}) \mid r\right\}
$$

Theorem 4.2. There is a basis for $E\left(\chi^{r \uparrow} \uparrow_{C}^{S_{n}}\right)$ indexed by $C$-orbits of length $n$ words with letters from $[\mathrm{m}]$ and with frequency dividing $r$. Moreover,

$$
\operatorname{ch}\left(\chi^{r \uparrow} \uparrow_{C}^{S_{n}}\right)=\operatorname{OFD}_{C, r}^{\text {cont }}(\mathbf{x})
$$

Proof. The proof of Theorem 3.1 goes through verbatim with the $C$-action replacing the $C_{n}$-action.

Our goal is broadly to replace $\operatorname{OFD}_{C, r}^{\text {cont }}(\mathbf{x})$ with a necklace generating function, apply cyclic sieving to get a major index generating function on words, and then apply RSK to get a Schur expansion.

Notation 4.3. For the rest of the section, suppose that $\sigma$ has disjoint cycle decomposition $\sigma=\sigma_{1} \cdots \sigma_{k}$ with $\nu_{i}:=\left|\sigma_{i}\right|$. Consequently, $\ell=|\langle\sigma\rangle|=\operatorname{lcm}\left(\nu_{1}, \ldots, \nu_{k}\right)$. Further, write

$$
C_{\nu}:=\left\{\sigma_{1}^{r_{1}} \cdots \sigma_{k}^{r_{k}} \in S_{n}: r_{1}, \ldots, r_{k} \in \mathbb{Z}\right\} \cong C_{\nu_{1}} \times \cdots \times C_{\nu_{k}}
$$

where $C_{\nu_{i}}:=\left\langle\sigma_{i}\right\rangle \subset S_{n}$. Thus, we have $C \subset C_{\nu} \subset S_{n}$.

In Section 3, we considered the $C_{n}$-orbits of $\mathrm{W}_{n}$, namely necklaces $N \in \mathrm{N}_{n}$. The frequency of $N$ is the stabilizer-order of $N$, i.e. freq $(N)=\# \operatorname{Stab}_{C_{n}}(N)$. We may group together $C_{n}$-orbits of $\mathrm{W}_{n}$ according to their stabilizer sizes by letting

$$
\mathrm{NF}_{n, r}:=\left\{N \in \mathrm{N}_{n}: \operatorname{freq}(N)=r\right\}
$$

be the set of necklaces of length $n$ words with frequency $r$. Similarly, $\mathrm{NFD}_{n, r}$ consists of $C_{n}$-orbits of $\mathrm{W}_{n}$ whose stabilizer is contained in the common stabilizer of $\mathrm{NF}_{n, r}$. 
Analogously, the $C_{\nu}$-orbits of $\mathrm{W}_{n}$ can be identified with products of necklaces $N_{1} \times$ $\cdots \times N_{k}$ or equivalently with tuples $\left(N_{1}, \ldots, N_{k}\right)$ where $N_{j} \in \mathrm{N}_{\nu_{j}}$. Since

$$
\operatorname{Stab}_{C_{\nu}}\left(N_{1} \times \cdots \times N_{k}\right)=\prod_{j=1}^{k} \operatorname{Stab}_{C_{\nu_{j}}}\left(N_{j}\right),
$$

we may group together $C_{\nu}$-orbits of $\mathrm{W}_{n}$ according to their stabilizers as follows.

Definition 4.4. Given $\nu=\left(\nu_{1}, \ldots, \nu_{k}\right)$ and $\rho=\left(\rho_{1}, \ldots, \rho_{k}\right)$, let

$$
\begin{aligned}
\mathrm{NF}_{\nu, \rho} & :=\mathrm{NF}_{\nu_{1}, \rho_{1}} \times \cdots \times \mathrm{NF}_{\nu_{k}, \rho_{k}}, \\
\mathrm{NFD}_{\nu, \rho} & :=\operatorname{NFD}_{\nu_{1}, \rho_{1}} \times \cdots \times \mathrm{NFD}_{\nu_{k}, \rho_{k}} .
\end{aligned}
$$

The elements of $\mathrm{NF}_{\nu, \rho}$ all have the same stabilizer, and the elements of $\mathrm{NFD}_{\nu, \rho}$ are precisely those whose stabilizer is contained in the common stabilizer of elements of $\mathrm{NF}_{\nu, \rho}$. We write $\rho \mid \nu$ to mean that $\rho_{i} \mid \nu_{i}$ for all $i=1, \ldots, r$. Note that $\mathrm{NF}_{\nu, \rho} \neq \varnothing$ if and only if $\rho \mid \nu$.

Given a group $G$ acting on a set $\mathrm{W}$ and a subgroup $H$ of $G$, each $G$-orbit of $\mathrm{W}$ is partitioned into $H$-orbits. Consequently, $C_{\nu}$-orbits of $\mathrm{W}_{n}$ are unions of $C$-orbits, which we exploit as follows.

Lemma 4.5. Let $\mathcal{O}$ be a $C$-orbit of $\mathrm{W}_{n}$. Let $N_{1} \times \cdots \times N_{k}$ be the $C_{\nu}$-orbit containing $\mathcal{O}$ and suppose $N_{1} \times \cdots \times N_{k} \in \mathrm{NF}_{\nu, \rho}$. Then

$$
\# \mathcal{O}=\operatorname{lcm}\left(\frac{\nu_{1}}{\rho_{1}}, \ldots, \frac{\nu_{k}}{\rho_{k}}\right)
$$

which depends only on $\nu$ and $\rho$. In particular,

$$
\mathcal{O} \in \mathrm{OFD}_{C, r} \quad \text { if and only if } \quad \ell \mid r \cdot \operatorname{lcm}\left(\frac{\nu_{1}}{\rho_{1}}, \ldots, \frac{\nu_{k}}{\rho_{k}}\right) .
$$

Proof. By assumption, $\operatorname{freq}\left(N_{j}\right)=\rho_{j}$ and $N_{j} \in \mathrm{N}_{\nu_{j}}$, so $\# N_{j}=\nu_{j} / \rho_{j}$. It follows that $\mathcal{O}$ is in bijection with the group generated by a permutation of cycle type $\left(\nu_{1} / \rho_{1}, \ldots, \nu_{k} / \rho_{k}\right)$, so that $\# \mathcal{O}=\operatorname{lcm}\left(\nu_{1} / \rho_{1}, \ldots, \nu_{k} / \rho_{k}\right)$. The second claim follows by noting that

$$
\mathcal{O} \in \mathrm{OFD}_{C, r} \Leftrightarrow \operatorname{freq}(\mathcal{O})|r \Leftrightarrow(\ell / \# \mathcal{O})| r \Leftrightarrow \ell \mid r \cdot \# \mathcal{O} .
$$

Lemma 4.6. We have

$$
\operatorname{OFD}_{C, r}^{\text {cont }}(\mathbf{x})=\sum \frac{\prod_{j=1}^{k} \frac{\nu_{j}}{\rho_{j}}}{\operatorname{lcm}\left(\frac{\nu_{1}}{\rho_{1}}, \ldots, \frac{\nu_{k}}{\rho_{k}}\right)} \operatorname{NF}_{\nu, \rho}^{\mathrm{cont}}(\mathbf{x}),
$$

where the sum is over all $\rho$ such that $\rho \mid \nu$ and $\ell \mid r \cdot \operatorname{lcm}\left(\frac{\nu_{1}}{\rho_{1}}, \ldots, \frac{\nu_{k}}{\rho_{k}}\right)$. 
Proof. Consider the map

$$
\Omega: \mathrm{OFD}_{C, r} \rightarrow \bigsqcup \mathrm{NF}_{\nu, \rho}
$$

sending $\mathcal{O} \in \mathrm{OFD}_{C, r}$ to the $C_{\nu}$-orbit containing $\mathcal{O}$, where the union is over all $\rho$ such that $\rho \mid \nu$ and $\ell \mid r \cdot \operatorname{lcm}\left(\frac{\nu_{1}}{\rho_{1}}, \ldots, \frac{\nu_{k}}{\rho_{k}}\right)$. By Lemma $4.5, \Omega$ does in fact map OFD $_{C, r}$ into this union, and $\Omega$ is surjective. Also, each $C_{\nu}$-orbit contained in $\mathrm{NF}_{\nu, \rho}$ has size $\operatorname{lcm}\left(\frac{\nu_{1}}{\rho_{1}}, \ldots, \frac{\nu_{k}}{\rho_{k}}\right)$, and $\# N_{1} \times \cdots \times N_{k}=\prod_{j=1}^{k} \frac{\nu_{j}}{\rho_{j}}$, so the fiber of each $N_{1} \times \cdots \times N_{k} \in \mathrm{NF}_{\nu, \rho}$ has size

$$
\# \Omega^{-1}\left(N_{1} \times \ldots \times N_{k}\right)=\frac{\prod_{j=1}^{k} \frac{\nu_{j}}{\rho_{j}}}{\operatorname{lcm}\left(\frac{\nu_{1}}{\rho_{1}}, \ldots, \frac{\nu_{k}}{\rho_{k}}\right)} .
$$

The result now follows from $\Omega$ being content-preserving.

In Section 3, we used cyclic sieving to turn generating functions involving $\operatorname{NFD}_{n, r}^{\text {cont }}(\mathbf{x})$ into Schur expansions. Thus our next goal is to turn the necklace generating function in Lemma 4.6 into an analogous generating function over $\operatorname{NFD}_{\nu, \rho}^{\text {cont }}(\mathbf{x})$. To accomplish this, one could in principle use Möbius inversion on the lattice of stabilizers of $C_{\nu}$-orbits to convert from $\mathrm{NF}_{\nu, \rho}^{\text {cont }}(\mathbf{x})$ to $\mathrm{NFD}_{\nu, \rho}^{\text {cont }}(\mathbf{x})$. However, the following argument is more direct.

Lemma 4.7. For $r=1, \ldots, \ell$,

$$
\operatorname{OFD}_{C, r}^{\text {cont }}(\mathbf{x})=\sum \operatorname{NFD}_{\nu, \tau}^{\text {cont }}(\mathbf{x})
$$

where the sum is over all $k$-tuples of integers $\tau \in\left[\nu_{1}\right] \times \cdots \times\left[\nu_{k}\right]$ such that $\sum_{j=1}^{k} \frac{\ell}{\nu_{j}} \tau_{j} \equiv_{\ell} r$. Proof. We have

$$
\mathrm{NFD}_{\nu, \tau}^{\mathrm{cont}}(\mathbf{x})=\sum_{\rho \mid \nu, \tau} \mathrm{NF}_{\nu, \rho}^{\mathrm{cont}}(\mathbf{x})
$$

where $\rho \mid \nu, \tau$ means $\rho_{j} \mid \nu_{j}$ and $\rho_{j} \mid \tau_{j}$ for all $j$. Consequently,

$$
\sum_{\substack{\tau \in\left[\nu_{1}\right] \times \cdots \times\left[\nu_{k}\right] \\ \sum_{j=1}^{k} \frac{\ell}{\nu_{j}} \tau_{j} \equiv \ell r}} \operatorname{NFD}_{\nu, \tau}^{\text {cont }}(\mathbf{x})=\sum_{\rho \mid \nu} c_{\nu, \rho}^{r} \mathrm{NF}_{\nu, \rho}^{\text {cont }}(\mathbf{x})
$$

where

$$
c_{\nu, \rho}^{r}:=\#\left\{\tau \in\left[\nu_{1}\right] \times \cdots \times\left[\nu_{k}\right]: \rho \mid \tau, \sum_{j=1}^{k} \frac{\ell}{\nu_{j}} \tau_{j} \equiv_{\ell} r\right\} .
$$

Since $\rho_{j} \mid \nu_{j}$ and $\rho_{j} \mid \tau_{j}$, write $\gamma_{j}:=\frac{\nu_{j}}{\rho_{j}} \in \mathbb{Z}_{\geqslant 1}$ and $\delta_{j}:=\frac{\tau_{j}}{\rho_{j}} \in \mathbb{Z}_{\geqslant 1}$. Then,

$$
\sum_{j=1}^{k} \frac{\ell}{\nu_{j}} \tau_{j}=\sum_{j=1}^{k} \frac{\ell}{\gamma_{j}} \delta_{j}
$$


SO

$$
c_{\nu, \rho}^{r}=\#\left\{\delta \in\left[\gamma_{1}\right] \times \cdots \times\left[\gamma_{k}\right]: \sum_{j=1}^{k} \frac{\ell}{\gamma_{j}} \delta_{j} \equiv_{\ell} r\right\} .
$$

Defining a group homomorphism

$$
\begin{gathered}
\phi: \prod_{i=1}^{k} \mathbb{Z} / \gamma_{j} \rightarrow \mathbb{Z} / \ell \\
\left(\delta_{1}, \ldots, \delta_{k}\right) \mapsto \sum_{j=1}^{k} \frac{\ell}{\gamma_{j}} \delta_{j},
\end{gathered}
$$

we now have $c_{\nu, \rho}^{r}=\# \phi^{-1}(r)$. Since $\frac{\ell}{\gamma_{1}} \mathbb{Z}+\cdots+\frac{\ell}{\gamma_{k}} \mathbb{Z}=\frac{\ell}{\operatorname{lcm}\left(\gamma_{1}, \ldots, \gamma_{k}\right)} \mathbb{Z}$, it follows that

$$
\begin{aligned}
\operatorname{im} \phi & =\left\{r \in \mathbb{Z} / \ell: \ell \mid r \cdot \operatorname{lcm}\left(\gamma_{1}, \ldots, \gamma_{k}\right)\right\}, \\
\# \operatorname{im} \phi & =\operatorname{lcm}\left(\gamma_{1}, \ldots, \gamma_{k}\right) .
\end{aligned}
$$

For $r \in \operatorname{im} \phi$, we then have

$$
c_{\nu, \rho}^{r}=\# \phi^{-1}(r)=\# \operatorname{ker} \phi=\frac{\gamma_{1} \cdots \gamma_{k}}{\operatorname{lcm}\left(\gamma_{1}, \ldots, \gamma_{k}\right)} .
$$

The result follows from Lemma 4.6.

Our next goal is to convert the necklace expansion in Lemma 4.7 into a Schur expansion. Recalling from Section 3 that $\mathrm{M}_{n, r}:=\left\{w \in \mathrm{W}_{n}: \operatorname{maj}_{n}(w)=r\right\}$, Lemma 3.2 tells us

$$
\operatorname{NFD}_{\nu, \tau}^{\text {cont }}(\mathbf{x})=\prod_{j=1}^{k} \operatorname{NFD}_{\nu_{j}, \tau_{j}}^{\text {cont }}(\mathbf{x})=\prod_{j=1}^{k} \mathrm{M}_{\nu_{j}, \tau_{j}}^{\text {cont }}(\mathbf{x}) .
$$

Interpreting the right-hand side of (34) in terms of words and comparing with the indexing set in Lemma 4.7 motivates the following variations on the major index.

Definition 4.8. Suppose $\nu \vDash n, \tau \in\left[\nu_{1}\right] \times \cdots \times\left[\nu_{k}\right]$, and $\ell=\operatorname{lcm}\left(\nu_{1}, \ldots, \nu_{k}\right)$. Let $\mathbf{m a j}_{\nu}: \mathrm{W}_{n} \rightarrow\left[\nu_{1}\right] \times \cdots \times\left[\nu_{k}\right]$ be defined as follows. For $w \in \mathrm{W}_{n}$, write $w=w^{1} \cdots w^{k}$ where each $w^{j}$ is a word in $\mathrm{W}_{\nu_{j}}$. Set

$$
\mathbf{m a j}_{\nu}(w):=\left(\operatorname{maj}_{\nu_{1}}\left(w^{1}\right), \ldots, \operatorname{maj}_{\nu_{k}}\left(w^{k}\right)\right) .
$$

Furthermore, let $\operatorname{maj}_{\nu}: \mathrm{W}_{n} \rightarrow[\ell]$ be defined by

$$
\operatorname{maj}_{\nu}(w):=\sum_{j=1}^{k} \frac{\ell}{\nu_{j}} \operatorname{maj}_{\nu}(w)_{j} \quad(\bmod \ell) .
$$

Consequently, we have $\operatorname{maj}_{(n)}=\operatorname{maj}_{n}$. Note that both $\mathbf{m a j}_{\nu}$ and $\mathrm{maj}_{\nu}$ are functions of $\operatorname{Des}(w)$. We may thus define both $\mathbf{m a j}_{\nu}$ and $\operatorname{maj}_{\nu}$ on $Q \in \operatorname{SYT}(n)$ using only $\operatorname{Des}(Q)$ in the same way. Equivalently, we may set $\mathbf{m a j}_{\nu}(Q):=\operatorname{maj}_{\nu}(w)$ and $\operatorname{maj}_{\nu}(Q):=\operatorname{maj}_{\nu}(w)$ for any $w$ such that $Q=Q(w)$. 
Example 4.9. Let $\nu=(5,3,3)$ and $w=44121361631$, so that $\ell=15, w_{1}=44121$, $w_{2}=361$, and $w_{3}=631$. We have

$$
\operatorname{maj}_{\nu}(w)=\left(\operatorname{maj}_{5}\left(w_{1}\right), \operatorname{maj}_{3}\left(w_{2}\right), \operatorname{maj}_{3}\left(w_{3}\right)\right)=(1,2,3)
$$

and, hence, $\operatorname{maj}_{\nu}(w)=\frac{15}{5} \cdot 1+\frac{15}{3} \cdot 2+\frac{15}{3} \cdot 3=13(\bmod 15)$.

Definition 4.10. Suppose $\nu \vDash n, \tau \in\left[\nu_{1}\right] \times \cdots \times\left[\nu_{k}\right]$. Let

$$
\mathrm{M}_{\nu, \tau}:=\left\{w \in \mathrm{W}_{n}: \mathbf{m a j}_{\nu}(w)=\tau\right\}
$$

Theorem 4.11. [Ste89, Theorem 3.3] Let $C$ be a cyclic subgroup of $S_{n}$ generated by an element of cycle type $\nu=\left(\nu_{1}, \ldots, \nu_{k}\right)$, and let $\ell=\operatorname{lcm}\left(\nu_{1}, \ldots, \nu_{k}\right)$. We have

$$
\sum_{r=1}^{\ell} \operatorname{ch}\left(\chi^{r \uparrow_{C}^{S_{n}}}\right) q^{r}=\mathrm{W}_{n}^{\text {cont, } \operatorname{maj}_{\nu}}(\mathbf{x} ; q)=\sum_{\substack{\lambda \vdash n \\ r \in[\ell]}} a_{\lambda, r}^{\nu} s_{\lambda}(\mathbf{x}) q^{r}
$$

where $a_{\lambda, r}^{\nu}:=\#\left\{Q \in \operatorname{SYT}(\lambda): \operatorname{maj}_{\nu}(Q)=r\right\}$. In particular, the multiplicity of $S^{\lambda}$ in $\chi^{r \uparrow S_{n}}$ is $a_{\lambda, r}^{\nu}$.

Proof. From the definition of $\mathbf{m a j}_{\nu}$ and Definition 4.4, we have

$$
\operatorname{NFD}_{\nu, \tau}^{\text {cont }}(\mathbf{x})=\mathrm{M}_{\nu, \tau}^{\text {cont }}(\mathbf{x}) \text {. }
$$

Using Theorem 4.2 and Lemma 4.7, we then have

$$
\begin{aligned}
\sum_{r=1}^{\ell} \operatorname{ch}\left(\chi_{C}^{r} \uparrow_{C}^{S_{n}}\right) q^{r} & =\sum_{r=1}^{\ell} \sum_{\substack{\tau \in\left[\nu_{1}\right] \times \cdots \times\left[\nu_{k}\right] \\
\sum_{j=1}^{k} \frac{\ell}{\nu_{j}} \tau_{j} \equiv_{\ell} r}} \operatorname{NFD}_{\nu, \tau}^{\text {cont }}(\mathbf{x}) q^{r} \\
& =\sum_{r=1}^{\ell} \sum_{\substack{\tau \in\left[\nu_{1}\right] \times \cdots \times\left[\nu_{k}\right] \\
\sum_{j=1}^{k} \frac{\ell}{\nu_{j}} \tau_{j} \equiv \ell}} \mathrm{M}_{\nu, \tau}^{\text {cont }}(\mathbf{x}) q^{r} \\
& =\sum_{r=1}^{\ell}\left\{w \in \mathrm{W}_{n}: \operatorname{maj}_{\nu}(w)=r\right\}^{\text {cont }}(\mathbf{x}) q^{r} \\
& =\mathrm{W}_{n}^{\text {cont, } \operatorname{maj}_{\nu}(\mathbf{x} ; q)}
\end{aligned}
$$

Since $\operatorname{maj}_{\nu}(w)$ depends only on $\operatorname{Des}(w)$, we can apply the RSK bijection again through Lemma 2.7 to get

$$
\mathrm{W}_{n}^{\text {cont }, \text { maj }_{\nu}}(\mathbf{x} ; q)=\sum_{\substack{\lambda \vdash n \\ r \in[\ell]}} a_{\lambda, r}^{\nu} s_{\lambda}(\mathbf{x}) q^{r}
$$


Remark 4.12. Stembridge showed the equality of the first and third terms in Theorem 4.11 using the skew analogue of (1) and branching rules along Young subgroups of $S_{n}$. By contrast, $\mathrm{W}_{n}^{\text {cont, } \operatorname{maj}_{\nu}}(\mathbf{x} ; q)$ played a key role in our approach.

Since the isomorphism type of $\chi^{r} \uparrow_{C}^{S_{n}}$, or equivalently the Schur expansion of $\operatorname{OFD}_{C, r}^{\text {cont }}(\mathbf{x})$, depends only on $\nu$, the cycle type of a generator of $C$, and $\operatorname{gcd}(\ell, r)$, we have the following generalization of Corollary 2.15.

Corollary 4.13. For all $n \geqslant 1$ and $\lambda, \nu \vdash n$, we have $a_{\lambda, r}^{\nu}=a_{\lambda, \operatorname{gcd}(\ell, r)}^{\nu}$, where $\ell=$ $\operatorname{lcm}\left(\nu_{1}, \nu_{2}, \ldots\right)$.

For use in the next section, we record the Schur expansion of $\mathrm{M}_{\nu, \tau}^{\mathrm{cont}}(\mathbf{x})$. The proof is analogous to the last step of the proof of Theorem 4.11 using Lemma 2.7.

Corollary 4.14. If $\nu, \tau \vDash n$, then

$$
\mathrm{M}_{\nu, \tau}^{\mathrm{cont}}(\mathbf{x})=\sum_{\lambda \vdash n} \mathbf{a}_{\lambda, \tau}^{\nu} s_{\lambda}(\mathbf{x})
$$

where

$$
\mathbf{a}_{\lambda, \tau}^{\nu}:=\#\left\{Q \in \operatorname{SYT}(\lambda): \mathbf{m a j}_{\nu}(Q)=\tau\right\} .
$$

We also have a corresponding symmetry result. Contrast it with Corollary 2.15.

Corollary 4.15. Suppose $\nu=\left(\nu_{1}, \ldots, \nu_{k}\right)$ is the cycle type of some $\sigma \in S_{n}, \tau \in\left[\nu_{1}\right] \times$ $\cdots \times\left[\nu_{k}\right], \pi \in S_{k}$, and $\lambda \vdash n$. Then, $\mathbf{a}_{\lambda, \tau}^{\nu}=\mathbf{a}_{\lambda, \pi \cdot \tau}^{\pi \cdot \nu}$.

Proof. Since reordering does not affect contents, we have

$$
\operatorname{NFD}_{\nu, \tau}^{\text {cont }}(\mathbf{x})=\operatorname{NFD}_{\pi \cdot \nu, \pi \cdot \tau}^{\text {cont }}(\mathbf{x}) \text {. }
$$

Now apply Corollary 4.14 and equate coefficients of $s_{\lambda}(\mathbf{x})$.

Remark 4.16. Finally, in the spirit of Section 3, we sketch how to reinterpret Theorem 4.11 as a cyclic sieving result for the maj $_{\nu}$ statistic. First define $f l e x_{\nu}: W_{n} \rightarrow[\ell]$ exactly as before, namely lexicographically order the $C$-orbit of $w \in \mathrm{W}_{n}$ to $\operatorname{compute} \operatorname{lex}_{\nu}(w)$ starting at 1 , let $\operatorname{freq}_{\nu}(w)$ denote the stabilizer-order of $w$ under the $C$-action, and let $\operatorname{flex}_{\nu}(w)=\operatorname{freq}_{\nu}(w) \cdot \operatorname{lex}_{\nu}(w)$. Exactly as in Lemma 3.2, each $\mathcal{O} \in \mathrm{OFD}_{n, r}$ contains a unique element with $\operatorname{flex}_{\nu}(w)=r$, so that Theorem 4.2 gives

$$
\sum_{r=1}^{\ell} \operatorname{ch} \chi^{r} \uparrow_{C_{n}}^{S_{n}} q^{r}=\sum_{r=1}^{\ell} \operatorname{OFD}_{n, r}^{\text {cont }}(\mathbf{x}) q^{r}=\mathrm{W}_{n}^{\text {cont }_{n} ; \operatorname{flex}_{\nu}}(\mathbf{x} ; q) .
$$

Using Theorem 4.11, it follows that

$$
\mathrm{W}_{n}^{\text {cont }_{f l e x}}(\mathbf{x} ; q)=\mathrm{W}_{n}^{\text {cont }_{\text {maj }}}(\mathbf{x} ; q) .
$$

Equivalently, $\operatorname{maj}_{\nu}$ and $\mathrm{flex}_{\nu}$ are equidistributed on each $\mathrm{W}_{\alpha}$. The analogue of the "universal" flex sieving result for $C_{n}$-actions on $\mathrm{W}_{n}$, Lemma 1.4, holds for $\operatorname{flex}_{\nu}$ and $C$ actions on $\mathrm{W}_{n}$ for the same straightforward reasons. Putting it all together, we find that $\left(\mathrm{W}_{\alpha}, C, \mathrm{~W}_{\alpha}^{\mathrm{maj}_{\nu}}(q)\right)$ exhibits the CSP. In this sense, $\mathrm{flex}_{\nu}, \mathrm{maj}_{\nu}$ are universal sieving statistics for the $C$-action on $\mathrm{W}_{n}$ on $C$-closed sets and $S_{n}$-closed sets, respectively, generalizing Lemma 1.4 and Corollary 1.3 and from the introduction. 


\section{Inducing 1-dimensional Representations from $C_{a} \prec S_{b}$ to $S_{a b}$}

We next apply the approach of Section 3 and Section 4 to prove a generalization of a formula due to Schocker [Sch03] for the Schur expansion of $\mathcal{L}_{\left(a^{b}\right)}$. In particular, we give Schur expansions of the characteristics of

$$
\mathcal{L}_{\left(a^{b}\right)}^{r, 1}:=\chi^{r, 1 \uparrow \uparrow_{C_{a} 2 S_{b}}^{S_{a b}}} \quad \text { and } \quad \mathcal{L}_{\left(a^{b}\right)}^{r, \epsilon}:=\chi^{r, \epsilon \uparrow_{C_{a} l S_{b}}^{S_{a b}}}
$$

Note that $\operatorname{ch} \mathcal{L}_{\left(a^{b}\right)}=\operatorname{ch} \mathcal{L}_{\left(a^{b}\right)}^{1,1}$ by Corollary 2.23 .

The argument in Corollary 2.23 and the fact that $\operatorname{ch}\left(\epsilon_{b}\right)=e_{b}(\mathbf{x})$ immediately yield the following more general result, which also follows from an appropriate modification of Theorem 3.1.

Lemma 5.1. We have

$$
\mathcal{L}_{\left(a^{b}\right)}^{r, 1}=\left(\left(\begin{array}{c}
\mathrm{NFD}_{a, r} \\
b
\end{array}\right)\right)^{\mathrm{cont}}(\mathbf{x}) \quad \text { and } \quad \mathcal{L}_{\left(a^{b}\right)}^{r, \epsilon}=\left(\begin{array}{c}
\mathrm{NFD}_{a, r} \\
b
\end{array}\right)^{\mathrm{cont}}(\mathbf{x}) .
$$

Our first goal is to manipulate the necklace generating functions in Lemma 5.1 in such a way that we may apply cyclic sieving. We use Burnside's lemma and a sign-reversing involution to unravel these multiset and subset generating functions, respectively.

Lemma 5.2. We have

$$
\left(\left(\begin{array}{c}
\mathrm{NFD}_{a, r} \\
b
\end{array}\right)\right)^{\mathrm{cont}}\left(x_{1}, x_{2}, \ldots\right)=\sum_{\nu \vdash b} \frac{1}{z_{\nu}} \prod_{j=1}^{\ell(\nu)} \mathrm{NFD}_{a, r}^{\mathrm{cont}}\left(x_{1}^{\nu_{j}}, x_{2}^{\nu_{j}}, \ldots\right) .
$$

Proof. Multisets of $b$ necklaces from $\mathrm{NFD}_{a, r}$ can be thought of as $S_{b}$-orbits of length- $b$ tuples $\left(N_{1}, \ldots, N_{b}\right)$ of necklaces $N_{i} \in \mathrm{NFD}_{a, r}$ under the natural $S_{b}$-action. The tuples $\left(N_{1}, \ldots, N_{b}\right)$ fixed by an element $\sigma \in S_{b}$ are those tuples which are constant on blocks corresponding to cycles of $\sigma$. It follows that if $\sigma$ has cycle type $\nu \vdash b$,

$$
\left\{T \in \mathrm{NFD}_{a, r}^{b}: \sigma \cdot T=T\right\}^{\operatorname{cont}}\left(x_{1}, x_{2}, \ldots\right)=\prod_{j=1}^{\ell(\nu)} \operatorname{NFD}_{a, r}^{\text {cont }}\left(x_{1}^{\nu_{j}}, x_{2}^{\nu_{j}}, \ldots\right) .
$$

By Burnside's lemma, we may count $S_{b}$-orbits of necklaces $\left(N_{1}, \ldots, N_{b}\right)$ of fixed content by averaging the number of $\sigma$-fixed tuples of fixed content over all $\sigma \in S_{b}$. The result follows by grouping together permutations of a given cycle type.

Lemma 5.3. We have

$$
\left(\begin{array}{c}
\mathrm{NFD}_{a, r} \\
b
\end{array}\right)^{\mathrm{cont}}(\mathbf{x})=\sum_{\nu \vdash b} \frac{(-1)^{b-\ell(\nu)}}{z_{\nu}} \prod_{j=1}^{\ell(\nu)} \operatorname{NFD}_{a, r}^{\mathrm{cont}}\left(x_{1}^{\nu_{j}}, x_{2}^{\nu_{j}}, \ldots\right) .
$$


Proof. Multiplying both sides by $b$ !, using (36) and the fact $\operatorname{sgn}(\sigma)=(-1)^{b-\ell(\nu)}$ for $\sigma \in S_{b}$ with cycle type $\nu$, the result is equivalent to

$$
\begin{aligned}
\left\{\left(N_{1}, \ldots, N_{b}\right)\right. & \left.\in \operatorname{NFD}_{a, r}^{b}:\left(N_{1}, \ldots, N_{b}\right) \text { are distinct }\right\}^{\text {cont }}(\mathbf{x}) \\
& =\sum_{\sigma \in S_{b}} \operatorname{sgn}(\sigma)\left\{T \in \operatorname{NFD}_{a, r}^{b}: \sigma \cdot T=T\right\}^{\text {cont }}(\mathbf{x}) .
\end{aligned}
$$

On the right-hand side of $(37)$, each $b$-tuple $\left(N_{1}, \ldots, N_{b}\right)$ is counted

$$
\operatorname{wt}\left(N_{1}, \ldots, N_{b}\right):=\sum_{\substack{\sigma \in S_{b} \\ \text { s.t. } \sigma \cdot\left(N_{1}, \ldots, N_{b}\right)=\left(N_{1}, \ldots, N_{b}\right)}} \operatorname{sgn}(\sigma)
$$

times. If $N_{1}, \ldots, N_{b}$ are distinct, then only $\sigma=\mathrm{id}$ contributes, $\operatorname{so} \operatorname{wt}\left(N_{1}, \ldots, N_{b}\right)=1$. If $N_{1}, \ldots, N_{b}$ are not distinct, then without loss of generality, suppose $N_{1}=N_{2}$. Then, modifying the cycle(s) containing 1 and 2 as in

$$
(1 \cdots)(2 \cdots) \leftrightarrow(1 \cdots 2 \cdots)
$$

gives a sign-reversing involution on $\left\{\sigma \in S_{b}: \sigma \cdot\left(N_{1}, \ldots, N_{b}\right)=\left(N_{1}, \ldots, N_{b}\right)\right\}$, meaning $\operatorname{wt}\left(N_{1}, \ldots, N_{b}\right)=0$. This proves $(37)$.

Remark 5.4. Using standard properties of plethysm (see e.g. [Mac95, §I.8])) and the power-sum expansions of $e_{b}$ and $h_{b}$ (see [Sta99, (7.22)-(7.23)]), Lemma 5.2 and Lemma 5.3 are equivalent to

$$
\begin{aligned}
\operatorname{ch} \mathcal{L}_{\left(a^{b}\right)}^{r, 1} & =h_{b}\left[\operatorname{ch} \chi^{r \uparrow{ }_{C_{a}}^{S_{a}}}\right]=\sum_{\nu \vdash b} \frac{1}{z_{\nu}} p_{\nu}\left[\operatorname{ch} \chi^{r \uparrow_{C_{a}}^{S_{a}}}\right], \\
\operatorname{ch} \mathcal{L}_{\left(a^{b}\right)}^{r, \epsilon} & =e_{b}\left[\operatorname{ch} \chi^{r \uparrow{ }_{C_{a}}^{S_{a}}}\right]=\sum_{\nu \vdash b} \frac{(-1)^{b-\ell(\nu)}}{z_{\nu}} p_{\nu}\left[\operatorname{ch} \chi^{r} \uparrow_{C_{a}}^{S_{a}}\right] .
\end{aligned}
$$

Consequently, one may replace the combinatorial manipulations in Lemma 5.2 and Lemma 5.3 with symmetric function manipulations. In the next section, we will prove Theorem 1.8, which generalizes the first equalities in (38) and (39).

Remark 5.5. Let $\omega$ be the involution on the algebra of symmetric functions defined by $\omega\left(s_{\lambda}(\mathbf{x})\right)=s_{\lambda^{\prime}}(\mathbf{x})$ where $\lambda^{\prime}$ is the conjugate of $\lambda$, obtained by reflecting $\lambda$ through the line $y=-x$. One may show in a variety of ways that

$$
\omega\left(\operatorname{ch} \chi^{r \uparrow S_{C_{n}}}\right)=\operatorname{ch} \chi^{s \uparrow S_{C_{n}}} \quad \text { where } \quad s=\left(\begin{array}{l}
n \\
2
\end{array}\right)-r .
$$

For instance, we can prove (40) using Theorem 1.6 as follows. Since conjugation $Q \mapsto Q^{\prime}$ satisfies $\operatorname{Des}\left(Q^{\prime}\right)=[n-1] \backslash \operatorname{Des}(Q)$, we have

$$
\begin{aligned}
a_{\lambda^{\prime}, r} & =\#\left\{Q \in \operatorname{SYT}\left(\lambda^{\prime}\right): \operatorname{maj}(Q) \equiv_{n} r\right\} \\
& =\#\left\{Q^{\prime} \in \operatorname{SYT}(\lambda): \operatorname{maj}\left(Q^{\prime}\right) \equiv_{n}\left(\begin{array}{l}
n \\
2
\end{array}\right)-r\right\}=a_{\lambda,\left(\begin{array}{l}
n \\
2
\end{array}\right)-r} .
\end{aligned}
$$


Therefore, by Theorem 1.6, letting $s=\left(\begin{array}{l}n \\ 2\end{array}\right)-r$,

$$
\omega\left(\operatorname{ch} \chi^{r} \uparrow_{C_{n}}^{S_{n}}\right)=\omega\left(\sum_{\lambda \vdash n} a_{\lambda, r} s_{\lambda}(\mathbf{x})\right)=\sum_{\lambda \vdash n} a_{\lambda^{\prime}, r} s_{\lambda}(\mathbf{x})=\sum_{\lambda \vdash n} a_{\lambda, s} s_{\lambda}(\mathbf{x})=\chi^{s \uparrow S_{C_{n}}} .
$$

From the symmetry result Corollary 2.15, it follows that $\operatorname{ch} \chi^{r \uparrow}{ }_{C_{n}}^{S_{n}}$ is fixed under $\omega$ when $n$ is odd. When $n$ is even, $\operatorname{ch} \chi^{r \uparrow S_{n}}$ may or may not be fixed. For instance, when $r=1$, we find

$$
\omega\left(\operatorname{ch} \mathcal{L}_{n}\right)=\omega\left(\operatorname{ch} \chi^{1 \uparrow C_{S_{n}}}\right)= \begin{cases}\operatorname{ch} \mathcal{L}_{n}^{(2)}=\operatorname{ch} \chi^{2 \uparrow T_{S_{n}}} & \text { if } n / 2 \text { is odd } \\ \operatorname{ch} \mathcal{L}_{n}=\operatorname{ch} \chi^{1 \uparrow_{S_{n}}^{C_{n}}} & \text { otherwise }\end{cases}
$$

Here $\mathcal{L}_{n}^{(2)}$ is the deformation of $\mathcal{L}_{n}$ recently studied by Sundaram [Sun18]. Further standard properties of plethysm together with (38) and (39) give

$$
\begin{aligned}
& \omega\left(\operatorname{ch} \mathcal{L}_{\left(a^{b}\right)}^{r, 1}\right)=\operatorname{ch} \mathcal{L}_{\left(a^{b}\right)}^{r, \epsilon} \quad \text { for } a \text { odd, and } \\
& \left.\begin{array}{l}
\omega\left(\operatorname{ch}_{\left(a^{b}\right)}^{r, 1}\right)=\operatorname{ch} \mathcal{L}_{\left(a^{b}\right)}^{s, 1} \\
\omega\left(\operatorname{ch}_{\left(a^{b}\right)}^{r, \epsilon}\right)=\operatorname{ch} \mathcal{L}_{\left(a^{b}\right)}^{s, \epsilon}
\end{array}\right\} \quad \text { for } a \text { even, where } s=\left(\begin{array}{l}
a \\
2
\end{array}\right)-r .
\end{aligned}
$$

Consequently, one may obtain the Schur expansion of $\operatorname{ch} \mathcal{L}_{\left(a^{b}\right)}^{r, \epsilon}$ from the Schur expansion of $\operatorname{ch} \mathcal{L}_{\left(a^{b}\right)}^{r, 1}$ simply by applying the $\omega$ map if and only if $a$ is odd. When $a$ is even, these two cases are more fundamentally different.

Next, we convert $\operatorname{NFD}_{a, r}^{\text {cont }}\left(x_{1}^{\nu_{j}}, x_{2}^{\nu_{j}}, \ldots\right)$ into a linear combination of $\mathrm{NF}_{k, s}^{\text {cont }}(\mathbf{x})$ 's and then apply Mobius inversion to convert to a linear combination of $\operatorname{NFD}_{k, s}^{\text {cont }}(\mathbf{x})$ 's. We will need the following variation on the number-theoretic Möbius function $\mu$.

Definition 5.6. Suppose $d \mid e$ and $f \mid e$. Set

$$
\mu_{f}(d, e):=\sum_{\substack{g \\ \text { s.t. } \operatorname{lcm}(f, d)|g| e}} \mu\left(\frac{g}{f}\right) .
$$

This expression simplifies considerably as follows. Let $\operatorname{rad}(m)$ denote the squarefree positive integer with the same prime divisors as $m$.

Lemma 5.7. Suppose $d \mid e$ and $f \mid e$. Then

$$
\mu_{f}(d, e)= \begin{cases}\mu\left(\frac{\operatorname{lcm}(f, d)}{f}\right) & \text { if } \operatorname{rad}\left(\frac{e}{f}\right)=\operatorname{rad}\left(\frac{\operatorname{lcm}(f, d)}{f}\right)=\frac{\operatorname{lcm}(f, d)}{f} \\ 0 & \text { otherwise. }\end{cases}
$$


Proof. We see

$$
\mu_{f}(d, e)=\sum_{\substack{g \\ \text { s.t. } \operatorname{lcm}(f, d)|g| e}} \mu\left(\frac{g}{f}\right)=\sum_{\substack{h \\ \text { s.t. } \frac{\operatorname{lcm}(f, d)}{f}|h| \frac{e}{f}}} \mu(h)
$$

Since $\mu(h) \neq 0$ only when $h$ is radical, $\mu(h) \neq 0$ only when $\frac{\operatorname{lcm}(f, d)}{f}$ is radical and $h \mid \operatorname{rad}(e / f)$. Restricting to this case, we can write $\operatorname{rad}(e / f)=k \operatorname{lcm}(f, d) / f$ for some integer $k$. Since $k$ and $\operatorname{lcm}(f, d) / f$ must be relatively prime, we have

$$
\begin{aligned}
\mu_{f}(d, e)= & \sum_{h} \mu(h) \\
& \text { s.t. } \frac{\operatorname{lcm}(f, d)}{f}|h| \frac{k \cdot \operatorname{lcm}(f, d)}{f} \\
& =\sum_{s \mid k} \mu\left(\left(\frac{\operatorname{lcm}(f, d)}{f}\right) s\right) \\
& =\mu\left(\frac{\operatorname{lcm}(f, d)}{f}\right) \sum_{s \mid k} \mu(s) \\
& = \begin{cases}\mu\left(\frac{\operatorname{lcm}(f, d)}{f}\right) & \text { if } k=1, \\
0 & \text { otherwise, }\end{cases}
\end{aligned}
$$

giving the result.

Lemma 5.8. We have

$$
\operatorname{NFD}_{a, r}^{\mathrm{cont}}\left(x_{1}^{k}, x_{2}^{k}, \ldots\right)=\sum_{s \mid r k} \mu_{s}(k, r k) \operatorname{NFD}_{a k, s}^{\mathrm{cont}}\left(x_{1}, x_{2}, \ldots\right) .
$$

Proof. The left-hand side is the content generating function for $k$-tuples of length $a$ necklaces with frequency dividing $r$ of the form $(N, \ldots, N)$, repeating the same necklace $k$ times. By concatenation, we may equivalently view such tuples as length $a k$ necklaces whose frequency $f$ satisfies $k|f| r k$. Consequently,

$$
\operatorname{NFD}_{a, r}^{\text {cont }}\left(x_{1}^{k}, x_{2}^{k}, \ldots\right)=\sum_{\substack{f \\ \text { s.t. } k|f| r k}} \operatorname{NF}_{a k, f}^{\text {cont }}\left(x_{1}, x_{2}, \ldots\right),
$$

recalling $\mathrm{NF}_{n, f}:=\left\{N \in \mathrm{N}_{n}:\right.$ freq $\left.(N)=f\right\}$. Applying Möbius inversion to the identity $\mathrm{NFD}_{a k, f}^{\text {cont }}(\mathbf{x})=\sum_{s \mid f} \mathrm{NF}_{a k, s}^{\mathrm{cont}}(\mathbf{x})$ gives

$$
\mathrm{NF}_{a k, f}^{\mathrm{cont}}(\mathbf{x})=\sum_{s \mid f} \mu\left(\frac{f}{s}\right) \mathrm{NFD}_{a k, s}^{\mathrm{cont}}(\mathbf{x}) .
$$


Thus, by (43), (42) becomes

$$
\begin{aligned}
\operatorname{NFD}_{a, r}^{\text {cont }}\left(x_{1}^{k}, x_{2}^{k}, \ldots\right) & =\sum_{\substack{f \\
\text { s.t. } k|f| r k}} \sum_{s \mid f} \mu\left(\frac{f}{s}\right) \operatorname{NFD}_{a k, s}^{\text {cont }}\left(x_{1}, x_{2}, \ldots\right) \\
& =\sum_{s \mid r k}\left(\sum_{\substack{f \\
\text { s.t. } \operatorname{lcm}(k, s)|f| r k}} \mu\left(\frac{f}{s}\right)\right) \operatorname{NFD}_{a k, s}^{\text {cont }}\left(x_{1}, x_{2}, \ldots\right) \\
& =\sum_{s \mid r k} \mu_{s}(k, r k) \operatorname{NFD}_{a k, s}^{\text {cont }}\left(x_{1}, x_{2}, \ldots\right) .
\end{aligned}
$$

by Definition 5.6.

Notation 5.9. Given a sequence $\nu=\left(\nu_{1}, \ldots, \nu_{k}\right) \in \mathbb{Z}_{\geqslant 1}^{k}$ and an integer $r \in \mathbb{Z}_{\geqslant 1}$, let

$$
r * \nu:=\left(r \nu_{1}, \ldots, r \nu_{k}\right) .
$$

Given another sequence $\tau=\left(\tau_{1}, \ldots, \tau_{k}\right)$, recall that $\tau \mid \nu$ means $\tau_{j} \mid \nu_{j}$ for all $j$. Further recall

$$
\mathrm{NFD}_{\nu, \tau}=\mathrm{NFD}_{\nu_{j}, \tau_{j}} \times \cdots \times \mathrm{NFD}_{\nu_{k}, \tau_{k}}
$$

from Definition 4.4. Finally, extend $\mu_{f}(d, e)$ to sequences multiplicatively:

$$
\mu_{\left(f_{1}, \ldots, f_{k}\right)}\left(\left(d_{1}, \ldots, d_{k}\right),\left(e_{1}, \ldots, e_{k}\right)\right):=\prod_{j=1}^{k} \mu_{f_{j}}\left(d_{j}, e_{j}\right) .
$$

Corollary 5.10. We have

$$
\begin{aligned}
\operatorname{ch} \mathcal{L}_{\left(a^{b}\right)}^{r, 1} & =\sum_{\nu \vdash b} \frac{1}{z_{\nu}} \sum_{\tau \mid r * \nu} \mu_{\tau}(\nu, r * \nu) \operatorname{NFD}_{a * \nu, \tau}^{\mathrm{cont}}(\mathbf{x}), \\
\operatorname{ch} \mathcal{L}_{\left(a^{b}\right)}^{r, \epsilon} & =\sum_{\nu \vdash b} \frac{(-1)^{b-\ell(\nu)}}{z_{\nu}} \sum_{\tau \mid r * \nu} \mu_{\tau}(\nu, r * \nu) \operatorname{NFD}_{a * \nu, \tau}^{\mathrm{cont}}(\mathbf{x}) .
\end{aligned}
$$

Proof. Combine Lemma 5.1, Lemma 5.2 or Lemma 5.3, and Lemma 5.8.

We may now state and generalize Schocker's formula for $\operatorname{ch} \mathcal{L}_{\left(a^{b}\right)}=\operatorname{ch} \mathcal{L}_{\left(a^{b}\right)}^{1,1}$.

Theorem 5.11 (See [Sch03, Thm. 3.1]). For all $a, b \geqslant 1$ and $r=1, \ldots, a$, we have

$$
\begin{aligned}
\operatorname{ch} \mathcal{L}_{\left(a^{b}\right)}^{r, 1} & =\sum_{\lambda \vdash a b}\left(\sum_{\nu \vdash b} \frac{1}{z_{\nu}} \sum_{\tau \mid r * \nu} \mu_{\tau}(\nu, r * \nu) \mathbf{a}_{\lambda, \tau}^{a * \nu}\right) s_{\lambda}(\mathbf{x}) \quad \text { and } \\
\operatorname{ch} \mathcal{L}_{\left(a^{b}\right)}^{r, \epsilon} & =\sum_{\lambda \vdash a b}\left(\sum_{\nu \vdash b} \frac{(-1)^{b-\ell(\nu)}}{z_{\nu}} \sum_{\tau \mid r * \nu} \mu_{\tau}(\nu, r * \nu) \mathbf{a}_{\lambda, \tau}^{a * \nu}\right) s_{\lambda}(\mathbf{x}),
\end{aligned}
$$

where, recalling the definition of $\mathbf{m a j}_{a * \nu}$ from Definition 4.8 ,

$$
\mathbf{a}_{\lambda, \tau}^{a * \nu}:=\#\left\{Q \in \operatorname{SYT}(\lambda): \mathbf{m a j}_{a * \nu}(Q)=\tau\right\} .
$$


Proof. Combine Corollary 4.14 and Corollary 5.10.

Remark 5.12. Schocker's approach to [Sch03, Thm. 3.1] is based on Jöllenbeck's noncommutative character theory and involved manipulations with Klyachko's idempotents and Ramanujan sums. Much of Schocker's argument generalizes immediately to all $r$. The argument presented above is comparatively self-contained and direct. Two perhaps mysterious aspects of the formula, the appearance of Möbius functions and the average over $S_{b}$, arose naturally from Burnside's lemma and a change of basis using Möbius inversion. Our argument uses explicit bijections at each step except for the appeal to Burnside's lemma and the use of Lemma 5.3.

\section{Higher Lie Modules and Branching Rules}

The argument in Section 3 solves Thrall's problem for $\lambda=(n)$ by considering all branching rules for $C_{n} \hookrightarrow S_{n}$ simultaneously and using cyclic sieving and RSK to convert from the monomial to the Schur basis. We now turn to analogous considerations for the higher Lie modules and more generally branching rules for $C_{a} 2 S_{b} \hookrightarrow S_{a b}$. We give an analogue of the flex statistic and the monomial basis expansion for such branching rules from Section 2.5. We then show how to convert from the monomial to the Schur basis assuming the existence of a certain statistic on words we call mash which interpolates between maj $_{n}$ and the shape under RSK.

We now recall and prove Theorem 1.8 from the introduction, after introducing some notation.

Definition 6.1. Fix integers $a, b \geqslant 1$. Define

$$
\mathrm{P}_{a}^{b}:=\left\{\underline{\lambda}=\left(\lambda^{(1)}, \ldots, \lambda^{(a)}\right): \lambda^{(1)}, \ldots, \lambda^{(a)} \text { are partitions }, \sum_{r=1}^{a}\left|\lambda^{(r)}\right|=b\right\},
$$

which indexes the irreducible $C_{a}$ \ $S_{b}$-representations by Theorem 2.20.

Theorem. For all $a, b \geqslant 1$ and $\underline{\lambda}=\left(\lambda^{(1)}, \ldots, \lambda^{(a)}\right) \in \mathrm{P}_{a}^{b}$, we have

$$
\operatorname{ch} S^{\lambda} \underline{\uparrow}_{C_{a} S_{b}}^{S_{a b}}=\prod_{r=1}^{a} s_{\lambda^{(r)}}\left[\operatorname{NFD}_{a, r}^{\mathrm{cont}}(\mathbf{x})\right] .
$$

Proof of Theorem 1.8. We have

$$
\begin{aligned}
& S^{\underline{\lambda} \uparrow}{ }_{C_{a} S_{b}}^{S_{a b}}=\left[\bigotimes_{r=1}^{a}\left(\chi_{a}^{r} 2 S^{\lambda^{(r)}}\right)\right] \uparrow_{C_{a} l S_{\alpha(\underline{\lambda})}}^{S_{a b}} \\
& \cong\left[\bigotimes_{r=1}^{a}\left(\chi_{a}^{r} 2 S^{\lambda^{(r)}}\right)\right] \uparrow_{T_{a} i S_{\alpha(\underline{\lambda})}}^{S_{a * \alpha(\lambda)}} S_{a * \alpha(\underline{\lambda})} \\
& \cong\left[\bigotimes_{r=1}^{a}\left(\chi_{a}^{r} 2 S^{\lambda^{(r)}}\right) \uparrow_{C_{a}\left\langle S_{\mid \lambda}(r)\right|}^{S_{a \mid \lambda(r)}}\right] \uparrow_{S_{a * \alpha(\underline{\lambda})}}^{S_{a b}}
\end{aligned}
$$




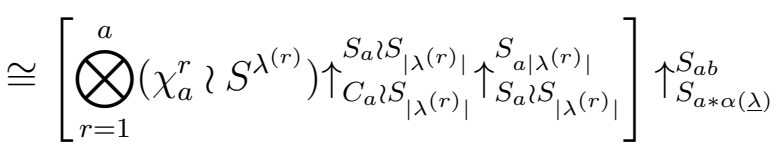

$$
\begin{aligned}
& \cong\left[\bigotimes_{r=1}^{a}\left(\chi_{a}^{r} \uparrow_{C_{a}}^{S_{a}} 2 S^{\lambda^{(r)}}\right) \uparrow_{S_{a}\left\langle S_{\mid \lambda}(r)\right|}^{S_{a|\lambda(r)|}}\right] \uparrow_{S_{a * \alpha(\underline{\lambda})}}^{S_{a b}},
\end{aligned}
$$

where the first and third isomorphisms use transitivity of induction, the second isomorphism uses Lemma 2.26, and the fourth isomorphism uses Lemma 2.25. Consequently, using (20), (22), and Theorem 3.1, we have

$$
\begin{aligned}
\operatorname{ch} S^{\underline{\lambda} \uparrow S_{C_{a} l S_{b}}} & =\prod_{r=1}^{a} \operatorname{ch}\left(\chi_{a}^{r} \uparrow_{C_{a}}^{S_{a}} 2 S^{\lambda^{(r)}}\right) \uparrow^{S_{a|\lambda(r)|}}{ }_{S_{a} S_{|\lambda\rangle}(r) \mid} \\
& =\prod_{r=1}^{a}\left(\operatorname{ch} S^{\lambda^{(r)}}\right)\left[\operatorname{ch} \chi_{a}^{r} \uparrow_{C_{a}}^{S_{a}}\right] \\
& =\prod_{r=1}^{a} s_{\lambda(r)}\left[\operatorname{NFD}_{a, r}^{\text {cont }}(\mathbf{x})\right] .
\end{aligned}
$$

Recall from Section 2.3 that given a word $w$, the shape of $w$, denoted $\operatorname{sh}(w)$, is the common shape of $P(w)$ and $Q(w)$ under RSK.

Definition 6.2. Fix $a, b \geqslant 1$. Construct statistics

$$
\mathrm{flex}_{a}^{b}, \mathrm{maj}_{a}^{b}: \mathrm{W}_{a b} \rightarrow \mathrm{P}_{a}^{b}
$$

as follows. Given $w \in \mathrm{W}_{a b}$, write $w=w^{1} \cdots w^{b}$ where $w^{j} \in \mathrm{W}_{a}$. In this way, consider $w$ as a word of size $b$ whose letters are in $\mathrm{W}_{a}$. For each $r \in[a]$, let $w^{(r)}$ denote the subword of $w$ whose letters are those $w^{j}$ such that $f \operatorname{ex}\left(w^{j}\right)=r$. Totally order $\mathrm{W}_{a}$ lexicographically, so that RSK is well-defined for words with letters from $\mathrm{W}_{a}$. Set

$$
\operatorname{flex}_{a}^{b}(w):=\left(\operatorname{sh}\left(w^{(1)}\right), \ldots, \operatorname{sh}\left(w^{(a)}\right)\right) .
$$

Define $\mathrm{maj}_{a}^{b}$ in the same way but with flex replaced by $\operatorname{maj}_{a}$. Consequently, $\operatorname{maj}_{n}^{1}(w)$ is the $n$-tuple of partitions whose only non-empty entry is a single cell at position maj ${ }_{n}(w)$.

Example 6.3. Let $w=212023101241$ and suppose $a=3, b=4$. Write

$$
w=(212)(023)(101)(241) .
$$

The parenthesized terms have flex statistics $2,1,2,2$ and maj $_{3}$ statistics $1,3,1,2$, respectively. When computing flex ${ }_{3}^{4}(w)$, we then have $w^{(1)}=(023), w^{(2)}=(212)(101)(241), w^{(3)}=$ $\varnothing$. Since $(101)<_{\operatorname{lex}}(212)<_{\operatorname{lex}}(241), \operatorname{sh}\left(w^{(2)}\right)=\operatorname{sh}(213)=(2,1)$. Consequently,

$$
\operatorname{flex}_{3}^{4}(212023101241)=((1),(2,1), \varnothing) .
$$

When computing $\operatorname{maj}_{3}^{4}(w)$, we have $w^{(1)}=(212)(101), w^{(2)}=(241), w^{(3)}=(023)$. Since $(101)<_{\operatorname{lex}}(212), \operatorname{sh}\left(w^{(1)}\right)=\operatorname{sh}(21)=(1,1)$. Hence

$$
\operatorname{maj}_{3}^{4}(212023101241)=((1,1),(1),(1)) .
$$


We now recall and prove Theorem 1.9 from the introduction.

Theorem. Fix $a, b \geqslant 1$. We have

$$
\begin{aligned}
& \sum_{\underline{\lambda} \in \mathrm{P}_{a}^{b}} \operatorname{dim} S^{\underline{\lambda}} \cdot \operatorname{ch}\left(S^{\underline{\lambda}} \uparrow_{C_{a} S_{b}}^{S_{a b}}\right) q^{\underline{\lambda}}=\mathrm{W}_{a b}^{\mathrm{cont}, \operatorname{flex}_{a}^{b}}(\mathbf{x} ; q) \\
& =\mathrm{W}_{a b}^{\mathrm{cont}, \mathrm{maj}_{a}^{b}}(\mathbf{x} ; q)
\end{aligned}
$$

where the $S^{\lambda}$ are irreducible representations of $C_{a} 2 S_{b}$ and the $q^{\underline{\lambda}}$ are independent indeterminates.

Proof of Theorem 1.9. Fix $\underline{\lambda} \in \mathrm{P}_{a}^{b}$. For the left-hand side, using Theorem 1.8 and (24),

$$
\operatorname{dim} S^{\lambda} \cdot \operatorname{ch}\left(\begin{array}{c}
S^{\lambda} \uparrow S_{a b} \\
C_{a} S_{b}
\end{array}\right)=\left(\begin{array}{c}
b \\
\alpha(\underline{\lambda})
\end{array}\right) \prod_{r=1}^{a} \# \operatorname{SYT}\left(\lambda^{(r)}\right) \cdot s_{\lambda^{(r)}}\left[\operatorname{NFD}_{a, r}^{\text {cont }}(\mathbf{x})\right] .
$$

For the right-hand side, we have

$$
\left.\mathrm{W}_{a b}^{\text {cont,flex }}{ }_{a}^{b}(\mathbf{x} ; q)\right|_{q^{\underline{\lambda}}}=\left\{w \in \mathrm{W}_{a b}: \operatorname{flex}_{a}^{b}(w)=\underline{\lambda}\right\}^{\operatorname{cont}}(\mathbf{x}) .
$$

Say $\alpha(\underline{\lambda})=\left(\alpha_{1}, \ldots, \alpha_{a}\right)$. In order for $w \in \mathrm{W}_{a b}$ to have $\operatorname{flex}_{a}^{b}(w)=\underline{\lambda}$, we must have $\operatorname{sh}\left(w^{(r)}\right)=\lambda^{(r)}$ for each $r \in[a]$. Recalling $\mathrm{F}_{a, r}:=\left\{w \in \mathrm{W}_{a}\right.$ : flex $\left.(w)=r\right\}$, we may thus choose each $w^{(r)} \in\left(\mathrm{F}_{a, r}\right)^{\alpha_{r}}$ with $\operatorname{sh}\left(w^{(r)}\right)=\lambda^{(r)}$ independently and then shuffle them in $\left(\begin{array}{c}b \\ \alpha(\underline{\lambda})\end{array}\right)$ ways to form $w$. Consequently,

$$
\begin{aligned}
& \left\{w \in \mathrm{W}_{a b}: \operatorname{flex}_{a}^{b}(w)=\underline{\lambda}\right\}^{\mathrm{cont}}(\mathbf{x}) \\
& \quad=\left(\begin{array}{c}
b \\
\alpha(\underline{\lambda})
\end{array}\right) \prod_{r=1}^{a}\left\{w^{(r)} \in\left(\mathrm{F}_{a, r}\right)^{\alpha_{r}}: \operatorname{sh}\left(w^{(r)}\right)=\lambda^{(r)}\right\}^{\operatorname{cont}}(\mathbf{x}) .
\end{aligned}
$$

The content generating function for words with a given shape $\mu \vdash n$ under RSK is given by

$$
\left\{w \in \mathrm{W}_{n}: \operatorname{sh}(w)=\mu\right\}^{\operatorname{cont}}(\mathbf{x})=\# \operatorname{SYT}(\mu) s_{\mu}(\mathbf{x}),
$$

since the number of possible $Q$-tableaux is $\# \mathrm{SYT}(\mu)$ and the content generating function for $P$-tableaux is $s_{\lambda}(\mathbf{x})$. Changing the alphabet from $\mathbb{Z}_{\geqslant 1}$ to $\mathrm{F}_{a, r}$ and using Lemma 3.2 gives

$$
\begin{aligned}
\left\{w^{(r)} \in\left(\mathrm{F}_{a, r}\right)^{\alpha_{r}}: \operatorname{sh}\left(w^{r}\right)=\lambda^{(r)}\right\}^{\operatorname{cont}}(\mathbf{x}) & =\# \operatorname{SYT}\left(\lambda^{(r)}\right) s_{\lambda^{(r)}}\left[\mathrm{F}_{a, r}^{\text {cont }}(\mathbf{x})\right] \\
& =\# \operatorname{SYT}\left(\lambda^{(r)}\right) s_{\lambda^{(r)}}\left[\operatorname{NFD}_{a, r}^{\text {cont }}(\mathbf{x})\right]
\end{aligned}
$$

The first equality in Theorem 1.9 now follows from combining (45) and (47) with (44). The second equality in Theorem 1.9 follows similarly. 
While Theorem 1.9 determines the monomial expansion of the graded Frobenius series tracking branching rules for $C_{a} \imath S_{b} \hookrightarrow S_{a b}$, we are ultimately interested in the corresponding Schur expansion. We next describe how the approach in the preceding sections might be used to find this Schur expansion. The key properties used in the proof of Theorem 1.6 converting from the monomial basis to the Schur basis were that maj ${ }_{n}$ is equidistributed with flex on each $\mathrm{W}_{\alpha}$ and $\operatorname{maj}_{n}(w)$ depends only on $Q(w)$. In order to apply a similar argument for $\operatorname{ch}\left(S{ }^{\lambda} \uparrow_{C_{a} l S_{b}}^{S_{a b}}\right)$, we need a statistic as follows.

Problem 6.4. Fix $a, b \geqslant 1$. Find a statistic

$$
\operatorname{mash}_{a}^{b}: \mathrm{W}_{a b} \rightarrow \mathrm{P}_{a}^{b}
$$

with the following properties.

(i) For all $\alpha \vDash a b, \operatorname{maj}_{a}^{b}$ (or equivalently $\mathrm{flex}_{a}^{b}$ ) and $\operatorname{mash}_{a}^{b}$ are equidistributed on $\mathrm{W}_{\alpha}$.

(ii) If $v, w \in \mathrm{W}_{a b}$ satisfy $Q(v)=Q(w)$, then $\operatorname{mash}_{a}^{b}(v)=\operatorname{mash}_{a}^{b}(w)$.

Finding such a statistic $\operatorname{mash}_{a}^{b}$ would determine the Schur decomposition of $\operatorname{ch}\left(S^{\lambda} \uparrow S_{a b} S_{a S_{b}}\right)$ as follows.

Corollary 6.5. Suppose $\operatorname{mash}_{a}^{b}$ satisfies Properties (i) and (ii) in Problem 6.4. Then

$$
\operatorname{ch}\left(S^{\underline{\lambda} \uparrow} S_{C_{a} S_{b}}\right)=\sum_{\nu \vdash a b} \frac{\#\left\{Q \in \operatorname{SYT}(\nu): \operatorname{mash}_{a}^{b}(Q)=\underline{\lambda}\right\}}{\operatorname{dim}(S \underline{\lambda})} s_{\nu}(\mathbf{x}),
$$

where $\operatorname{mash}_{a}^{b}(Q):=\operatorname{mash}_{a}^{b}(w)$ for any $w \in \mathrm{W}_{a b}$ with $Q(w)=Q$.

Proof. We use, in order, Theorem 1.9, Property (i), RSK, and Property (ii) to compute

$$
\begin{aligned}
& \left.\sum_{\underline{\lambda} \in \mathrm{P}_{a}^{b}} \operatorname{dim}\left(S^{\underline{\lambda}}\right) \operatorname{ch}\left(S^{\underline{\lambda}} \uparrow_{C_{a} S_{b}}^{S_{a b}}\right)\right) q^{\underline{\lambda}}=\mathrm{W}_{a b}^{\mathrm{cont}, \mathrm{maj}_{a}^{b}}(\mathbf{x} ; q) \\
& =\sum_{\alpha \models a b} \mathrm{~W}_{\alpha}^{\operatorname{maj}_{a}^{b}}(q) \mathbf{x}^{\alpha} \\
& =\sum_{\alpha \models a b} \mathrm{~W}_{\alpha}^{\operatorname{mash}_{a}^{b}}(q) \mathbf{x}^{\alpha} \\
& =\mathrm{W}_{a b}^{\mathrm{cont}, \operatorname{mash}_{a}^{b}}(\mathbf{x} ; q) \\
& =\sum_{\nu \vdash a b}(\operatorname{SSYT}(\nu) \times \operatorname{SYT}(\nu))^{\operatorname{cont} \operatorname{mash}_{a}^{b}}(\mathbf{x} ; q) \\
& =\sum_{\nu \vdash a b} \operatorname{SSYT}(\nu)^{\operatorname{cont}}(\mathbf{x}) \operatorname{SYT}(\nu)^{\operatorname{mash}_{a}^{b}}(q) \\
& =\sum_{\nu \vdash a b} \operatorname{SYT}(\nu)^{\operatorname{mash}_{a}^{b}}(q) s_{\nu}(\mathbf{x}) .
\end{aligned}
$$

The result follows by equating coefficients of $q^{\lambda}$. 
Remark 6.6. When $a=1$ and $b=n$, we may replace $\underline{\lambda}$ with $\lambda \vdash n$. Under this identification, $\operatorname{maj}_{1}^{n}(w)=\operatorname{sh}(w)$, which clearly satisfies Properties (i) and (ii). When $a=n$ and $b=1$, we may replace $\underline{\lambda}$ with an element $r \in[n]$. Under this identification, we may set $\operatorname{mash}_{n}^{1}(w)=\operatorname{maj}_{n}(w)$, which satisfies Properties (i) and (ii). In this sense $\operatorname{mash}_{a}^{b}$ interpolates between the major index $\operatorname{maj}_{n}$ and the shape under RSK, hence the name.

While maj $_{a}^{b}$ trivially satisfies Property (i), it fails Property (ii) already when $a=b=2$, as in the following example.

Example 6.7. Let $v=2314$ and $w=1423$. Then,

$$
Q(v)=Q(w)=\begin{array}{|l|l|l|}
\hline 1 & 2 & 4 \\
\hline 3 &
\end{array}
$$

while

$$
\begin{aligned}
\operatorname{maj}_{2}^{2}(v) & =(\varnothing,(1,1)) \\
\operatorname{maj}_{2}^{2}(w) & =(\varnothing,(2)) .
\end{aligned}
$$

Remark 6.8. When defining $\operatorname{flex}_{a}^{b}$ and $\mathrm{maj}_{a}^{b}$, we somewhat arbitrarily chose the lexicographic order on $\mathrm{W}_{a}$. Any other total order would work just as well. However, maj $\mathrm{j}_{a}^{b}$ continues to fail Property (ii) using any other total order when $a=b=2$ in Example 6.7 since either $14<23$ or $23<14$.

\section{Acknowledgements}

We would like to heartily thank our advisor, Sara Billey, for helpful discussions and extensive comments on the manuscript. We would also like to thank Vic Reiner, Christophe Reutenauer, Brendon Rhoades, John Stembridge, and Sheila Sundaram for helpful conversations on this material and related topics.

\section{References}

[AS18] C. Ahlbach and J. P. Swanson. Refined cyclic sieving on words for the major index statistic. European J. Combin., 73:37-60, 2018.

[BBG90] F. Bergeron, N. Bergeron, and A. M. Garsia. Idempotents for the free Lie algebra and q-enumeration. In Invariant theory and tableaux (Minneapolis, MN, 1988), volume 19 of IMA Vol. Math. Appl., pages 166-190. Springer, New York, 1990.

[BER11] A. Berget, S.-P. Eu, and V. Reiner. Constructions for cyclic sieving phenomena. SIAM J. Discrete Math., 25(3):1297-1314, 2011.

[Bra44] A. Brandt. The free Lie ring and Lie representations of the full linear group. Trans. Amer. Math. Soc., 56:528-536, 1944.

[CFL58] K.-T. Chen, R. H. Fox, and R. C. Lyndon. Free differential calculus, IV. The quotient groups of the lower central series. Ann. of Math. (2), 68:81-95, 1958. 
[Fu197] W. Fulton. Young Tableaux, volume 35 of London Mathematical Society Student Texts. Cambridge University Press, Cambridge, 1997.

[Gar90] A. M. Garsia. Combinatorics of the free Lie algebra and the symmetric group. In Analysis, et cetera, pages 309-382. Academic Press, Boston, MA, 1990.

[GP92] A. M. Garsia and C. Procesi. On certain graded $S_{n}$-modules and the $q$-Kostka polynomials. Adv. Math., 94(1):82-138, 1992.

[GR93] I. M. Gessel and C. Reutenauer. Counting permutations with given cycle structure and descent set. J. Combin. Theory Ser. A, 64(2):189-215, 1993.

[Hal59] M. Hall, Jr. The Theory of Groups. The Macmillan Co., New York, N.Y., 1959.

[Kly74] A. A. Klyachko. Lie elements in the tensor algebra. Siberian Mathematical Journal, 15(6):914-920, 1974.

[KW01] W. Kraśkiewicz and J. Weyman. Algebra of coinvariants and the action of a Coxeter element. Bayreuth. Math. Schr., (63):265-284, 2001.

[LLT94] A. Lascoux, B. Leclerc, and J.-Y. Thibon. Green polynomials and HallLittlewood functions at roots of unity. European J. Combin., 15(2):173-180, 1994.

[LLT97] A. Lascoux, B. Leclerc, and J.-Y. Thibon. Ribbon tableaux, Hall-Littlewood functions, quantum affine algebras, and unipotent varieties. J. Math. Phys., 38(2):1041-1068, 1997.

[Mac13] P. A. MacMahon. The indices of permutations and the derivation therefrom of functions of a single variable associated with the permutations of any assemblage of objects. Amer. J. Math., 35(3):281-322, 1913.

[Mac95] I. G. Macdonald. Symmetric functions and Hall polynomials. Oxford Mathematical Monographs. The Clarendon Press, Oxford University Press, New York, second edition, 1995.

[Rei15] V. Reiner. Thrall's problem and coarsenings. Banff workshop on positivity in algebraic combinatorics lecture slides, 2015.

[Reu93] C. Reutenauer. Free Lie Algebras, volume 7 of London Mathematical Society Monographs. New Series. The Clarendon Press, Oxford University Press, New York, 1993.

[Rho10] B. Rhoades. Hall-Littlewood polynomials and fixed point enumeration. Discrete Math., 310(4):869-876, 2010.

[RSW04] V. Reiner, D. Stanton, and D. White. The cyclic sieving phenomenon. J. Combin. Theory Ser. A, 108(1):17-50, 2004.

[Sag01] B. E. Sagan. The Symmetric Group, volume 203 of Graduate Texts in Mathematics. Springer-Verlag, New York, second edition, 2001.

[Sag11] B. E. Sagan. The cyclic sieving phenomenon: a survey. In Surveys in Combinatorics 2011, volume 392 of London Math. Soc. Lecture Note Ser., pages 183-233. Cambridge Univ. Press, Cambridge, 2011. 
[Sch63] M. P. Schützenberger. Quelques remarques sur une construction de Schensted. Math. Scand., 12:117-128, 1963.

[Sch03] M. Schocker. Multiplicities of higher Lie characters. J. Aust. Math. Soc., 75(1):9-21, 2003.

[Spe32] W. Specht. Eine Verallgemeinerung der symmetrischen Gruppe. PhD thesis, Humboldt-Universität zu Berlin, null, 1932.

[Spr74] T. A. Springer. Regular elements of finite reflection groups. Invent. Math., 25:159-198, 1974.

[Sta99] R. P. Stanley. Enumerative Combinatorics, Volume 2, volume 62 of Cambridge Studies in Advanced Mathematics. Cambridge University Press, Cambridge, 1999.

[Ste89] J. R. Stembridge. On the eigenvalues of representations of reflection groups and wreath products. Pacific J. Math., 140(2):353-396, 1989.

[Sun94] S. Sundaram. The homology representations of the symmetric group on CohenMacaulay subposets of the partition lattice. Adv. Math., 104(2):225-296, 1994.

[Sun18] S. Sundaram. Variations on the $S_{n}$-module $\operatorname{Lie}_{n}, 2018$ arXiv:1803.09368.

[Thr42] R. M. Thrall. On symmetrized Kronecker powers and the structure of the free Lie ring. Amer. J. Math., 64:371-388, 1942. 